\title{
Cellular mechanisms of heterogeneity in NF2-mutant schwannoma
}

Christine Chiasson-MacKenzie ${ }^{1,2}$, Ching-Hui Liu' ${ }^{1,2}$, Jeremie Vitte ${ }^{3}$, Elizabeth A. Flynn ${ }^{1,4}$,

Shannon L. Stott ${ }^{1,4}$, Marco Giovannini ${ }^{3}$ and Andrea I. McClatchey ${ }^{1,2^{*}}$

${ }^{1}$ Massachusetts General Hospital Cancer Center, Harvard Medical School, $14913^{\text {th }}$ Street, Charlestown, MA 02129, USA

${ }^{2}$ Department of Pathology, Massachusetts General Hospital, Harvard Medical School, 55 Fruit

Street, Boston, Massachusetts, 02114, USA

${ }^{3}$ Department of Head and Neck Surgery, David Geffen School of Medicine at UCLA and Jonsson Comprehensive Cancer Center (JCCC), University of California Los Angeles, Los Angeles, CA 90095, USA

${ }^{4}$ Center for Engineering in Medicine and BioMEMS Resource Center, Surgical Services, Massachusetts General Hospital, Harvard Medical School, $11416^{\text {th }}$ Street, Charlestown, Massachusetts, 02129, USA

*Correspondence: mcclatch@helix.mgh.harvard.edu 


\begin{abstract}
Schwannomas are common sporadic nervous system tumors and are diagnostic features of familial neurofibromatosis type 2 (NF2) that develop predominantly on cranial and spinal nerves and cause severe neurological deficits and significant morbidity. Virtually all schwannomas result from inactivation of the NF2 tumor suppressor gene with few, if any, cooperating mutations. Despite their genetic uniformity schwannomas exhibit remarkable clinical and therapeutic heterogeneity, which has impeded the success of early rational therapies. An understanding of how heterogeneity develops in NF2-mutant schwannomas is critically needed to improve therapeutic options for these patients. We have found that loss of the membrane:actin cytoskeleton-associated NF2 tumor suppressor protein, merlin, yields unstable intrinsic polarity and enables $\mathrm{Nf}^{-/-}$Schwann cells to adopt distinct programs of coordinated ErbB ligand production and polarized signaling, suggesting a self-generated model of schwannoma heterogeneity. We validated the heterogeneous distribution of biomarkers of these programs in schwannoma tissue and cell lines from well-established mouse models, and then took advantage of the synchronous development of multiple lesions in a mouse model to establish a quantitative pipeline that can be used to study how schwannoma heterogeneity evolves and impacts nerve function and therapeutic response in mouse, and ultimately human schwannomas.
\end{abstract}

\title{
Introduction
}

Schwannomas account for a large proportion of sporadic nervous system tumors in humans and are hallmarks of the inherited tumor predisposition syndrome neurofibromatosis type $2(\mathrm{NF} 2)^{1-4}$. Although usually benign, schwannomas develop predominantly on and around spinal and cranial nerves, causing significant neurological deficit, chronic pain and morbidity. Few targeted therapies have been developed to treat schwannomas and instead, high-risk 
surgical removal is often necessary. This is particularly difficult for familial NF2 patients, who frequently develop multiple, recurring tumors.

Whether inherited or sporadic, virtually all schwannomas are caused by genetic inactivation of the NF2 tumor suppressor gene, and few cooperating mutations have been identified $^{5}$. Despite being genetically 'cold', schwannomas exhibit surprising heterogeneity, including remarkably variable natural histories, growth rates, histological features and clinical impact ${ }^{1}$. Neither growth rate nor tumor burden correlates with the ability of schwannomas to cause pain or nerve dysfunction, and the few rational therapies that have been tested for schwannoma have yielded at best a heterogeneous and cytostatic response, often followed by accelerated tumor regrowth after drug cessation ${ }^{6-8}$. An understanding of the molecular basis of schwannoma heterogeneity is essential for the development of successful non-surgical therapies.

Schwannoma heterogeneity is also evident histologically and ultrastructurally ${ }^{9-11}$. Hematoxylin- and eosin-staining of schwannoma tissue reveals regions of spindle-shaped cells that form concentric whorls or aligned arrays, and others of more radially-shaped cells with shorter cell processes. Ultrastructurally, cells within schwannomas exhibit striking variation in the extent of cell-cell versus cell-basal lamina contact, with apposing cell surfaces coated with dense basal lamina or forming interlocking cell-cell contacts in widely varying proportions, and some regions featuring denuded basal lamina, prominent vacuoles and microcysts ${ }^{9}$. Such 'Antoni $A$ and B' regions exhibit different patterns of immune infiltration and vascularity, suggesting that they harbor distinct paracrine environments ${ }^{12}$. It is not known how these visible features of cellular heterogeneity are established within otherwise genetically homogeneous tumors.

Schwann cells (SCs) are uniquely polarized epithelial cells that can transition between a bipolar, migratory state during development and wound repair, and an apicobasally polarized state that relies on heterotypic contact with the neuron cell body (soma) or axon as an extrinsic 
spatial polarity cue ${ }^{13-15}$. Rather than the discrete junctional and fluid-exposed apical surfaces of conventional epithelia, apicobasally polarized SCs form a hybrid 'apicojunctional' adaxonal surface that is dedicated to contacting the neuron, enriched in both apical and cell-cell adhesion proteins, and a crucial site of metabolic communication between the two cell types ${ }^{16-19}{ }^{20}$. The remaining abaxonal surface contacts basal lamina that is generated by the SCs themselves. Importantly, signaling at the two SC surfaces is distinct ${ }^{14}$. For example, neuregulin $1(\mathrm{Nrg} 1)$ that is tethered to the neuronal plasma membrane activates ErbB3 on the adaxonal SC surface to induce the growth of axon-ensheathing membrane and mammalian target of rapamycin complex 1 (mTORC1)-activated protein translation at the expanding membrane edge, while laminin signaling through $\alpha 6$-containing integrins promotes mTORC2-dependent activation of serum and glucocorticoid-induced kinases (SGKs) abaxonally ${ }^{14,21-23}$. Notably, laminin-activated feedback limits Nrg1-ErbB3-driven membrane production at the adaxonal surface, suggesting that the size of two signaling domains is normally under feedback control via unknown mechanisms ${ }^{21}$. In vivo SC polarity is initiated and spatially defined by contact with the neuronal cell membrane but in vitro it is imposed and sized by attachment to a laminin-coated culture dish. In schwannomas, the spatial cues that normally govern polarized signaling are lost, and cells exhibit strikingly variable polarized surface content.

The NF2-encoded tumor suppressor, merlin, and closely related plasma membrane:cytoskeleton linking ERM proteins (ezrin, radixin, moesin) share interdependent roles in organizing the cell cortex and govern intrinsic cell polarity in many contexts ${ }^{24-31}$. For example, in single matrix-embedded epithelial cells, merlin-deficiency impairs the spatial restriction of cortical ezrin that normally governs intrinsic polarity; instead, ectopic cortical ezrin drives unstable intrinsic polarity ${ }^{27}$. Consistent with such fundamental cell cortex-organizing activities, merlin and the ERMs influence many signaling programs, including the activity of small GTPases of the Rho and Ras families, the Hippo signaling network and mTOR kinases ${ }^{32-}$ 36. Merlin/ERM proteins also modulate the surface availability and activity of a number of 
membrane receptors, and specifically the trafficking of and cytoskeletal response to members of the ErbB family of tyrosine kinases ${ }^{37-43}$. In liver epithelial cells merlin deficiency enhances the excitability of the cortical cytoskeleton to Epidermal Growth Factor (EGF) stimulation in an ezrindependent manner, enabling macropinocytosis, an actin-based mechanism of nutrient scavenging that is initiated by large cell surface ruffles that engulf extracellular fluid ${ }^{38,44}$. Thus, through fundamental roles in organizing the cell cortex, merlin and the ERM proteins are poised to govern the coordination of intrinsic polarity with other fundamental cellular activities.

We have found that NF2-deficient SCs exhibit unstable polarity and can adopt distinct phenotypic states that feature autocrine ErbB ligand and polarity gene expression and polarized cytoskeletal organization. Our data suggest a novel model of self-generated heterogeneity that could explain the notoriously variable clinical and therapeutic behaviors of schwannomas. Importantly, this model also suggests new biomarkers of schwannoma heterogeneity that we have validated in vivo using a quantitative imaging pipeline that we have developed. These findings provide mechanistic insight into the adaptive biology of SCs, new biomarkers and a quantitative imaging platform that can be developed to guide treatment strategy for human schwannoma.

\section{Results}

\section{Nf2-deficient SCs exhibit altered intrinsic polarity and exaggerated cortical responses to}

\section{Nrg1}

When cultured on laminin-coated tissue culture dishes, Nf $2^{\text {flox/flox }}$ (WT) SCs are bipolar with small ruffles forming at the poles, reflecting intrinsic polarity that is independent of cues imposed by axonal contact (Fig. 1A,B). In contrast, three different primary adult mouse Nf2 floxfllox SC populations in which Nf2 has been acutely deleted (hereafter denoted $N f 2^{--/}$; Supplemental Fig. 1A) exhibited a loss of this intrinsic polarity, instead featuring multiple dynamically extending processes containing larger ruffles, as has been reported (Fig. 1A,B) ${ }^{45}$. Multipolarity 
in $\mathrm{Nf}^{-/-} \mathrm{SCs}$ is accompanied by a more radial cell shape and distribution of actin, compared to the long parallel stress fibers exhibited by bipolar WT SCs (Fig. 1C; Supplemental Fig. 1B). This is consistent with the fundamental role for merlin in establishing intrinsic cortical polarity that has been observed in many cell types ${ }^{27,29}$.

Nrg1 is a major regulator of SC proliferation, migration, and myelination in vivo ${ }^{21,46}$. When $\mathrm{Nf}^{-/-}$SCs were acutely stimulated with $\mathrm{Nrg} 1$, we observed a dramatic reorganization of actin to form a circumferential band from which large cortical ruffles emanated. In contrast, WT SCs exhibit only weak cortical ruffles in response to Nrg1, despite activating similar levels of downstream signals (Fig. 1C,D; Supplemental Fig. 1C,D). On the other hand, when control and $\mathrm{Nf}^{-/-} \mathrm{SCs}$ were deprived of Nrg, which is normally included in SC culture medium, basally positioned microvilli were enhanced; in control cells microvilli were restricted largely to bipolar cell ends but, like Nrg1-induced cortical ruffles, were radially distributed in $\mathrm{Nf}^{-/-}$SCs (Fig. 1C,E; Supplemental Fig. 1E,F). Ezrin was relocalized to cortical ruffles or microvilli in the presence or absence of Nrg1, weakly in WT and dramatically in $\mathrm{Nf2}^{-/-} \mathrm{SCs}$ (Fig. 1E,F). Indeed, depletion of ezrin eliminated both multipolarity and the exaggerated cytoskeletal responses to Nrg1 availability in $\mathrm{Nf}^{-/-} \mathrm{SCs}$, consistent with a model wherein ectopic cortical ezrin drives the exaggerated formation of cortical structures in $\mathrm{Nf}^{-/-} \mathrm{SCs}$ as in other cell types (Fig. 1G; Supplemental Fig. $1 \mathrm{G}-\mathrm{I})^{38}$. Together these data reveal that in $\mathrm{Nf}^{-/-} \mathrm{SCs}$ multipolarity is associated with distinct and exaggerated cytoskeletal responses to the presence (ruffles) or absence (microvilli) of Nrg1.

\section{Progressive junctional ruffling, signaling and adhesion in $\mathrm{Nf2}^{-/-} \mathrm{SCs}$ in the presence of}

\section{Nrg1}

Like the axon-facing 'apicojunctional' surface of normal SCs in vivo, cortical ruffles in Nrg1-stimulated WT and $\mathrm{Nf}^{-/-}$SCs contain the adherens junction proteins $\mathrm{N}$-cadherin and $\beta$ - 
catenin, as well as the Nrg1 receptor ErbB3 and its heterodimeric partner ErbB2 (Fig. 2A-B) ${ }^{48-50}$. In fact, we observed a stronger enrichment of ErbB2, ErbB3, and the key ErbB3 effector pAkt in Nrg1-stimulated ruffles in Nf2/- SCs compared to WT SCs, despite similar levels of total ErbB3, pAkt and pERK (Fig. 2C-F; Supplemental Fig. 1D). Moreover, in contrast to WT SCs that restrict $\mathrm{N}$-cadherin-based cell-cell contacts to bipolar cell tips, $\mathrm{Nf}^{-/-} \mathrm{SCs}$ make contacts around their entire periphery, from which cortical ruffles emerge upon Nrg1-stimulation (Fig. 2A,B). Indeed, WT SCs did not adhere to one another and, instead undergo contact inhibition of locomotion, but ruffling membranes in $\mathrm{Nf}^{-/-} \mathrm{SCs}$ are 'sticky', enabling the formation of durable intercellular adhesions (Fig. 2G,H; Supplemental Video 1 and 2) ) $^{51}$ Importantly, this phenotype is progressive; at late confluence $\mathrm{Nf2}^{-/-} \mathrm{SCs}$ exhibit continuous junctional ruffling and form tightly packed aggregates that eventually lose attachment to the laminin-coated culture dish (Fig. 2I, Supplemental Fig. 2A). In contrast, at late confluence WT SCs round up as individual cells that do not adhere to one another (Supplemental Fig. 2A). Notably, WT and Nf2-- SCs express similar levels of $\mathrm{N}$-cadherin and of Sox2, which can induce relocalization of $\mathrm{N}$-cadherin to SC contacts, and little E-cadherin (Supplemental Fig. 2B,C) ${ }^{50}$, suggesting that Nf2-deficiency does not drive increased adhesion by these mechanisms. Thus, in the presence of Nrg1 Nf2deficiency enables the progressive expansion of an adhesive 'apicojunctional' ErbB signaling compartment.

Growth factor stimulated cortical actin ruffles can trigger macropinocytosis, a form of extracellular nutrient engulfment that is a signature of Nf2-deficiency in other cell types ${ }^{38,44}$. Indeed, we found that Nrg1-stimulated $\mathrm{Nf}^{-/-}$but not WT SCs exhibit uptake of the macropinocytic cargo dextran-488 that is blocked by the macropinocytosis inhibitor 5-(N-ethyl$\mathrm{N}$-isopropyl) amiloride (EIPA) (Fig. 3A-C). In real time, macropinosomes could readily be seen internalizing from ruffling cell-cell contacts (Supplemental Video 3). Schwannoma cells derived from genetically engineered Nf2-mutant mice also exhibited striking Nrg1-stimulated ruffling and macropinocytosis that was reversed by $N f 2$ re-expression or ezrin depletion (Fig. 3D,E; 
Supplemental Fig. 3A-I). Importantly, complementary in vivo and ex vivo approaches revealed prominent macropinocytosis that could be blocked by EIPA occurring in schwannomas arising in the well-established Postn-Cre;Nf2 floxflox mouse model ${ }^{52}$ (Fig. 3F-H). This observation could have immediate therapeutic ramifications as it is increasingly appreciated that large macrotherapeutics gain entry into cells via macropinocytosis ${ }^{53}$. As one example, extracellular vesicles (EVs) can be engineered to deliver many therapeutic cargoes, including nucleic acids for gene replacement ${ }^{54-56}$. Indeed, we found that $N f 2^{-/-}$schwannoma cells readily take up labelled EVs in an EIPA-sensitive manner (Fig. 3I). Thus, in the presence of Nrg1 exaggerated junctional cortical ruffling by $\mathrm{Nf2}^{-/-} \mathrm{SCs}$ can trigger macropinocytosis and tumor-selective uptake of macrotherapeutics.

\section{$\mathrm{Nf2}^{-/-}$SCs develop autocrine heterogeneity}

In vivo, the unrestricted expansion of cell-cell contact, junctional Nrg1-signaling and macropinocytosis exhibited by cultured $\mathrm{Nf}^{-/-} \mathrm{SCs}$ would occur when intercellular contact is permitted and Nrg1 is available. However, normal SCs strictly limit cell-cell contact and cells within schwannomas do not retain axonal contact and must therefore adapt to the loss of axonally provided Nrg1. We reasoned that $\mathrm{Nf2}^{-/-}$SCs might adapt by producing their own Nrg1, like other tumors that deploy autocrine ErbB ligand expression to induce cortical ruffling and macropinocytic scavenging ${ }^{57}$. Therefore, we asked whether Nrg1 deprivation similarly triggers autocrine signaling in $\mathrm{Nf}^{-/-} \mathrm{SCs}$. We found that Nrg1 deprivation yielded a modest but statistically significant increase in Nrg1 expression in both WT and $\mathrm{Nf}^{-/-} \mathrm{SCs}$ but caused a dramatic upregulation of the EGFR-specific ligands Egf and Tgfa specifically in Nf2/- SCs (Fig. 4A; Fig. 5B). Surprisingly however, although EGF ligands trigger cortical ruffles and macropinocytosis in many cell types, including $N f 2^{-/-}$liver epithelial cells ${ }^{38,57}$, both EGF and TGF $\alpha$ suppress these phenotypes in $N f 2^{-/}$SCs, and instead enhance the formation of basal actin stress fibers and the distribution of ezrin to basal microvilli and adjacent junctions (Fig. 4B- 
D, Supplemental Fig. 4A $)^{47}$. Moreover, Nrg1-deprived $N 2^{-/-}$SCs also upregulated multiple components of the SC basal signaling platform, including laminin (Lama2) and the laminin receptors $\alpha 6$ integrin (Itga6) and $\alpha$-dystroglycan (Dag1) (Fig. 4E) ${ }^{6,7,14,58}$. The accompanying increase in laminin and $\alpha 6$ integrin proteins is consistent with the enhanced basal cytoskeletal organization seen under conditions of Nrg-deprivation (Supplemental Fig. 4B). Both Nrgdeprived WT and $\mathrm{Nf}^{-/-}$SCs also upregulated Egfr, which is normally expressed at low levels in SCs but is increased in some schwannomas, some of which respond to pharmacologic EGFR inhibition (Supplemental Fig. 4C) ${ }^{6}$. However, WT SCs neither upregulate EGF ligands nor ruffle in response to them (Fig. 4A; Supplemental Fig. 4D). Thus, Nf2-- SCs may adapt to Nrg1deprivation by upregulating an EGFR-associated basal polarity program.

In other tumors, autocrine ruffling and macropinocytosis are strongly induced upon glutamine depletion ${ }^{57}$. Indeed, we found that in $\mathrm{Nf}^{-/-}$SCs glutamine depletion strongly exacerbated ruffling and macropinocytosis that is blocked by the pan-ErbB inhibitor afatinib (ErbBi; Fig. 5A; Supplemental Fig. 5 A,B). Consistent with the ability of Nrg1 but not EGF or TGF $\alpha$ to induce ruffling, the expression of $N r g 1$, specifically the soluble isoform, but not TGFa is dramatically enhanced under these conditions (Fig. 5B; Supplemental Fig. 5C,D). Moreover, conditioned medium from glutamine-deprived but not glutamine-replete $\mathrm{Nf}^{-/-} \mathrm{SCs}$ induced membrane ruffling and EIPA- and ErbBi-sensitive macropinocytosis in $\mathrm{Nf}^{-/-}$SCs (Fig. 5C-E). In contrast, conditioned medium from Nrg1-deprived $\mathrm{Nf2}^{-/-} \mathrm{SCs}$, like recombinant TGF $\alpha$ or EGF, enhanced basal structures (Supplementary Fig. 5E). Notably, Nrg1 upregulation itself was blocked by ErbBi treatment, suggesting a feedforward mechanism of autocrine Nrg1 production (Fig. 5F). Finally, the upregulation of Nrg1 in response to glutamine deprivation was completely rescued by the addition of exogenous glutamate (Fig. 5G). This is important given that peripheral nerves themselves may be a key source of glutamate that fuels SCs through a glutamate-glutamine cycle as in the central nervous system ${ }^{59,60}$. Thus, SCs likely interpret 
glutamine deficiency as loss of axonally produced glutamate. Together these data suggest that Nf2-deficient SCs can activate either an autocrine Nrg1-driven apicojunctional program of signaling and macropinocytic scavenging or an autocrine EGF-ligand driven basal program in the absence of nutrients normally provided by axons.

\section{Heterogeneous mTOR activation and drug sensitivity}

In normal SCs in vivo axonal Nrg1 drives the polarized activation of mTORC1, as measured by phospho-S6 ribosomal protein (pS6) levels ${ }^{14}$. Aberrant mTORC1 signaling has been implicated in schwannoma, but the pharmacologic mTOR inhibitor everolimus (mTORi), one of the only drug-based therapies being evaluated in schwannoma patients, has yielded variable and cytostatic results thus far, which could reflect a heterogeneous tumordependency ${ }^{34,35,61}$. Our studies suggest that pS6 may be a specific biomarker of autocrine Nrg1 production in schwannoma. Indeed, we found that pS6 levels were elevated specifically in nutrient-starved Nrg1-expressing $\mathrm{Nf}^{-/-}$but not WT SCs, and treatment of $\mathrm{Nf2}^{-/-} \mathrm{SCs}$ with conditioned medium from glutamine-deprived $\mathrm{Nf2}^{-/-} \mathrm{SCs}$ dramatically stimulated pS6 while medium from Nrg1-deprived, TGF $\alpha$-expressing $\mathrm{Nf}^{-/-}$SCs actually lowered pS6 levels (Fig. 6AC). Consistently, Nrg1, TGF $\alpha$ and pS6 were each heterogeneously distributed across schwannoma lesions in vivo, and the majority of pS6+ cells also expressed high levels of Nrg1 (Fig. 6D,E). Importantly, however, we found that high Nrg1-expressing $N 2^{-/-}$SCs are resistant to mTORi relative to $\mathrm{Nrg} 1$-deprived, TGF $\alpha$-expressing $\mathrm{Nf}^{-/-} \mathrm{SCs}$, and mTORi has no impact on Nrg1 expression (Fig. 6F,G). On the other hand, ErbB inhibition blocks Nrg1 but not TGF $\alpha$ expression (Fig. 6H). These results could explain the cytostatic and heterogeneous clinical efficacy of mTORi, as well as the 'rebound' tumor growth that is often seen upon cessation of mTORi treatment and could be triggered by a reservoir of accumulated Nrg1. Overall, these 
data have important ramifications for designing and interpreting therapeutic strategies for schwannoma.

\section{Self-generated heterogeneity}

Collectively, our observations suggest that when deprived of axonal signals, $\mathrm{Nf2}^{-/-} \mathrm{SCs}$ can adopt two distinct programs of coordinated ligand production and cytoskeletal polarity: High Nrg1 expression and apicojunctional polarity dominated by cortical actin ruffling and macropinocytosis, or high TGF $\alpha$ expression and a basal polarity program dominated by basal actin stress fibers and ECM receptors. Wild-type SCs exhibit weak fluctuations in the same gene expression programs and are relatively impervious to ligand-induced changes in cytoskeletal polarity (Fig. 7A,B). The bipotential, ligand-associated and progressive features of $\mathrm{Nf}^{-/-}$SC phenotypes could enable self-generated heterogeneity within schwannomas. To begin to test this, we examined clonal populations of $\mathrm{Nf2}^{-/-} \mathrm{SCs}$ by limiting dilution. Indeed, we found that individual clones exhibited either predominantly basal cytoskeletal polarity, high Tgfa and Egf expression, low pS6 and low dextran-488 uptake, or apicojunctional polarity, high Nrg1 expression and high dextran-488 uptake (Fig 7C-E; Supplemental Fig. 6A-F). Mouse schwannoma cell lines also exhibited features of one or the other of these distinct programs, with cells from one schwannoma (schwannoma A) exhibiting strong sensitivity to basal program induction and a strong signaling response to TGF $\alpha$, and those from another (schwannoma B) exhibiting strong Nrg1-induced signaling and sensitivity to apicojunctional program induction, but insensitivity to Nrg1 deprivation (Fig. 7F; Supplemental Fig. 6G). Importantly, although the character of individual clonal SC lines was biased toward one program or the other, heterogeneity was still evident at a cellular level (Supplemental Fig 6D), supporting the idea that Nf2-deficiency enables this self-generated bipotential heterogeneity. In fact, examination of TGF $\alpha$ distribution in parental $\mathrm{Nf}^{-/-}$SCs subjected to short-term Nrg1 deprivation, a condition in 
which both Nrg1 and TGF $\alpha$ mRNA upregulation is detected in the bulk population, versus a clonal line subjected to longterm Nrg1 deprivation, revealed a transition from scattered highly TGF $\alpha$-expressing cells at early timepoints to large clusters of TGF $\alpha$-expressing cells within a bed of TGF $\alpha$-negative cells at later timepoints, providing a glimpse into how self-generated heterogeneity may evolve (Fig. 7G).

\section{Quantitative analysis of self-generated heterogeneity in schwannoma}

To begin to analyze the development of schwannoma heterogeneity in vivo, we took advantage of the synchronous development of schwannomas in each of the 15 dorsal root ganglia (DRG) of the Postn-Cre;Nf2 floxflox mouse model between 3-6 months of age. This model provides a unique opportunity to monitor the evolution of both intra- and inter-tumoral heterogeneity. We developed a quantitative pipeline to study schwannoma heterogeneity by immunofluorescence-based antibody staining of formalin-fixed paraffin-embedded (FFPE) sections, whole slide scanning of all DRGs, and digital image analysis using HALO ${ }^{\mathrm{TM}}$ software (Indica Labs). A digital image analysis algorithm was developed to detect Nrg1 and TGF $\alpha$ in DRG lesions in a 6 month old Postn-Cre;Nf flox/flox mouse at single-cell resolution and a Random Forest machine learning algorithm was applied to exclude large DRG soma. This analysis revealed intralesion heterogeneity for both Nrg1 and TGF $\alpha$ at this early timepoint, and particularly for Nrg1+ cells, a clear spatial bias adjacent to individual DRG soma (Fig. 8 A,B; Supplemental Fig. 7A). While the percentage of Nrg1+ cells in each lesion appeared to vary considerably, this variability was largely eliminated when normalized to the area of DRG soma in each lesion, both suggesting minimal interlesional heterogeneity at this early stage and supporting the discovery of a biased distribution of Nrg1+ cells near nerve soma (Fig $8 \mathrm{C}$ ). Indeed, spatial profiling using the proximity analysis algorithm revealed that a large percentage of $\mathrm{Nrg} 1+$ cells resided within a $25 \mu \mathrm{M}$ radius of each DRG soma (Fig. $8 \mathrm{C}$ ). To monitor 
heterogeneity as SC lesions expand, we compared DRG lesions in 6- and 12-month old PostnCre;Nf2 ${ }^{10 x / l o x}$ mice. While the percentage of Nrg1+ and TGF $\alpha+$ cells, and spatial bias of Nrg1+ cells remained similar, we noted a clear shift in the spatial distribution of TGF $\alpha+$ cells in some lesions at 12 months. Instead of individual and small clusters of TGF $\alpha+$ cells that are present in 6 month lesions, at 12 months TGF $\alpha+$ cells often formed large clustered arrays, particularly at the lesion edge (Fig. 8D). Indeed, aggregation of TGF $\alpha+$ cells in a subset of 12 month lesions could be readily captured by HALO-assisted classifier training and analysis of mean cluster size (Fig. 8E). Together these data identify biomarkers of a novel model of self-generated heterogeneity in early schwannoma lesions and provide insight into the evolution of schwannoma heterogeneity using a novel quantitative pipeline.

\section{Discussion}

Clinical and therapeutic heterogeneity is a major issue in schwannoma despite the unique genetic homogeneity of these common tumors. Indeed, it is currently not possible to predict schwannoma growth rate, impact on nerve function, or therapeutic response, rendering clinical management difficult. While much current focus in other cancers is on tumor:microenvironment interactions as drivers of heterogeneity in advanced tumors, little is known about intrinsic mechanisms of tumor heterogeneity. The genetic simplicity of schwannomas provides a unique window into this important aspect of tumorigenesis and the self-generating design principle we propose here likely also applies to more genetically complex tumors, particularly epithelial cancers that variably exhibit features of polarized cell architecture. Our studies therefore provide broader insight into the contribution of intrinsic mechanisms to tumor heterogeneity.

Our studies point to the ability of $\mathrm{Nf2}^{-/-} \mathrm{SCs}$ to enact distinct programs of ErbB ligand production and cytoskeletal polarity upon loss of axonally-provided nutrients as a key driver of 
schwannoma heterogeneity and suggest a model wherein this bipotentiality is triggered by unstable intrinsic cortical polarity. In vivo intrinsic SC polarity is stabilized by axonal contact that spatially limits the SC apicojunctional surface. Unstable polarity driven by the failure to limit cortical ezrin could facilitate loss of axonal contact, inappropriate cell-cell contact, and stochastic differences in the inheritance of cortical material upon division as occurs in other $\mathrm{Nf}^{-}$ 1- epithelial cell types ${ }^{27}$. Heterogeneity could be further biased by the amount of cell-cell versus cell-basal lamina contact experienced by schwannoma cells as they divide and disconnect from the axon and by juxtacrine exposure to ErbB ligands from neighboring cells. Ultimately, the recruitment of other cell types that are known to be heterogeneously present in schwannomas may contribute ${ }^{62}$. Our model would be consistent with the variable activation of multiple signaling pathways in $\mathrm{Nf}^{-/-} \mathrm{SCs}$, including mTORC1, mTORC2 and YAP/TAZ ${ }^{22,34}$. Indeed, we found that the SC YAP/TAZ targets Itga6 and Dag1 are elevated in Nf2-- SCs only upon Nrg1deprivation, which hyperactivates the basal polarity program, and in a basal program-biased schwannoma cell line, which would be consistent with the well-known activation of YAP/TAZ in response to cell-ECM attachment (Fig. 4E, 6A) ${ }^{63,64}$.

In addition to explaining the ultrastructural and histological features of schwannomas, distinct metabolic and signaling programs associated with each polarized 'state' could underlie the heterogeneous clinical behavior and therapeutic response of schwannomas. For example, glial cells and nerves provide reciprocal metabolic support to each other in the CNS and PNS, and metabolic dysfunction in glial cells is known to cause neurodegeneration ${ }^{15,21}$. The striking macropinocytic scavenging associated specifically with apicojunctional but not basal programs of $\mathrm{Nf2}^{-/-} \mathrm{SCs}$ in vitro suggests distinct metabolic programming, which could differentially impact adjacent nerves. Our work also suggests that tumors and/or tumor regions with significant macropinocytic activity will be particularly amenable to macrotherapeutic modes of delivery. On the other hand, differential signaling triggered by the two polarity states could confer the heterogeneous therapeutic responsiveness seen for mTORC1- and EGFR-inhibiting drugs in 
schwannoma patients ${ }^{6,8}$. While this may suggest combination therapies, it will be important to determine whether heterogeneity within schwannomas is stable, or whether $\mathrm{Nf}^{-/-} \mathrm{SCs}$ adapt to signaling blockades, perhaps by reverting to the other polarized state. Indeed, our discovery that pS6 is a biomarker of Nrg1 expression but not of mTORi sensitivity in $\mathrm{Nf}^{-/-} \mathrm{SCs}$, together with the observation that mTORi does not block Nrg1 expression itself, speaks to the complex but clinically relevant circuitry that drives the development of these genetically simple tumors. It is interesting to note that autocrine Nrg1 expression has been recently reported in association with NF2-mutant meningioma, but in this case, is blocked by mTORi, also underscoring the celltype specific biologies that govern such signaling circuitry ${ }^{65}$.

In the context of a schwannoma, unstable polarity will manifest three dimensionally in association with nerves, and a deeper mechanistic understanding of how heterogeneity develops and evolves requires a way to monitor it in situ. The unique availability of a mouse model in which multiple Nf2-mutant schwannomas develop synchronously in anatomically defined locations provides an opportunity to begin to test the model suggested by our in vitro studies and to monitor the evolution of heterogeneity as tumors develop and expand. By imaging and quantitatively analyzing all of the lesions in a single mouse, we captured the power of this model to both confirm and extend aspects of our in vitro studies. In addition to validating Nrg1 and TGF $\alpha$ as biomarkers of schwannoma heterogeneity, we found a spatial bias of Nrg1expressing cells within lesions in proximity to DRG soma, a source of Nrg1 that could drive the feedforward nature of Nrg1 expression that we see in vitro. Notably, it has been reported that neuronal Nrg1 levels are reduced by NF2 haploinsufficiency in some nerves, which could further contribute to heterogeneity in NF2 patients ${ }^{43}$. Similarly, the progressive clustering of TGF $\alpha+$ cells in developing lesions may mirror the expansion of TGF $\alpha+$ cell clusters upon Nrg1deprivation in vitro. These studies set the stage for a deeper analysis of how tumor heterogeneity evolves over longer periods of time in the mouse model, and a critical comparison 
to human schwannomas. It will be particularly interesting to examine markers of stromal, endothelial and immune cell types to define the timing of their recruitment to and spatial distribution within expanding schwannomas. Additional biomarkers can be multiplexed to create multivariate 'heterogeneity indices' for schwannoma that could be mapped to both tumor behavior (ie growth rate or nerve dysfunction) and therapeutic response, with the ultimate goals of predicting tumor behavior, guiding treatment strategy and improving therapeutic options for sporadic schwannoma and particularly NF2 patients, for whom few options exist.

\section{Materials and Methods}

\section{Mice}

The mouse schwannoma $A$ and $B$ cell lines were derived from tumors dissected from $P 0$ CreB; $N 2^{\text {flox/flox }}$ and $P 0-S C H-\Delta(39-121)-27$ mice, respectively ${ }^{66,67}$. The genetically engineered mouse model Postn-Cre;Nf2 floxflox of NF2 schwannomas was generated by breeding Postn-Cre and $N f 2^{f l o x}$ mice ${ }^{66,68}$. All mouse strains were maintained on FVB/N genetic background. Mice were monitored twice a week for 24 months or until a tumor or evidence of a tumor (paralysis, swelling, lethargy) was observed. All animal care and experimentation were performed with the approval of the UCLA Institutional Animal Care and Use Committees under protocol number 2019-042.

\section{Cell culture and reagents}

Primary murine SCs were isolated from sciatic nerves of $N f 2^{\text {floxfllox }}$ adult mice and purified by magnetic sorting as previously described ${ }^{66,69}$. SCs were routinely cultured in N2 medium (DMEM/F12-HAM, 1x N2 supplement (Thermo Fisher), 50 g/ml gentamicin (Thermo Fisher), 2 $\mu \mathrm{M}$ forskolin (Calbiochem) and $10 \mathrm{ng} / \mathrm{ml} \mathrm{Nrg} 1$ (HRG-beta-1 EGF domain) (R\&D Systems) on poly-L-lysine $(50 \mu \mathrm{g} / \mathrm{ml}$, Millipore Sigma) and mouse laminin $(10 \mu \mathrm{g} / \mathrm{ml}$, Thermo Fisher) coated plates and incubated at $37^{\circ} \mathrm{C}$ and $7.5 \% \mathrm{CO}_{2} . \mathrm{Nf2}$ was deleted from $\mathrm{Nf} 2^{\text {flox/flox }} \mathrm{SCs}$ via 
adenovirus infection with Cre-recombinase (Ad5-CMV-Cre). Clonal populations of $\mathrm{Nf}^{-/-} \mathrm{SCs}$ were generated by culturing cells in the absence of Nrg1 for at least 14 days and serial dilution to obtain single cell clones. Surviving subclones were maintained in Nrg1 free media. Tumoral SCs from mouse schwannomas were isolated by dispase/collagenase dissociation as described $^{69}$ and cultured in DMEM supplemented with $10 \%$ fetal bovine serum (FBS), 1x N2 supplement, $50 \mu \mathrm{g} / \mathrm{ml}$ gentamicin, $2 \mu \mathrm{M}$ forskolin, and $10 \mathrm{ng} / \mathrm{ml} \mathrm{Nrg} 1 \mathrm{on}$ poly-L-lysine and laminin coated plates at $37^{\circ} \mathrm{C}$ and $7.5 \% \mathrm{CO}_{2}$. Adenovirus infection was used for $\mathrm{Nf} 2 \mathrm{re}-$ expression (Ad5-CMV-Nf2 ${ }^{W T}$ ). 293A cells for adenovirus production (Agilent), and 293T cells for lentivirus production (ATCC) were cultured in 10\% FBS-DMEM with 1\% penicillin/streptomycin (Thermo Fisher) and incubated at $37^{\circ} \mathrm{C}$ and $5 \% \mathrm{CO}_{2}$.

\section{Growth factors and reagents}

Nrg was used at $10 \mathrm{ng} / \mathrm{ml}$; All other growth factors were obtained from Peprotech. EGF was used at $10 \mathrm{ng} / \mathrm{ml}$; TGF $\alpha$ was used at $10 \mathrm{ng} / \mathrm{ml}$; HGF was used at $50 \mathrm{ng} / \mathrm{ml}$; PDGF was used at $50 \mathrm{ng} / \mathrm{ml}$. Drug pretreatment was as follows: EIPA (Millipore Sigma), $50 \mu \mathrm{M}, 60 \mathrm{~min}$; afatanib (Selleck), $2 \mu \mathrm{M}, 24$ hours (h). These doses were maintained throughout the experiment.

\section{Plasmids and shRNA constructs}

The $N f 2^{W T}$ expression construct was generated by PCR amplification of the mouse Nf2 coding region and cloned into a pAdCMV vector as described ${ }^{37}$. The shRNA constructs targeting mouse ezrin (5'-ATTTCCTTGTTATAATCTCCG-3') in a pLKO-puro.1 vector from GE Healthcare and described in ${ }^{27}$. The control (shScr; (5'-CAGTCGCGTTTGCGACTGG-3') in a pLKO-puro. 1 vector was provided by Marianne James (MGH, Boston $)^{70}$.

\section{Virus production and infection}


$N f 2^{W T}$-expressing and Cre-recombinase adenoviruses were generated using the AdEasy system (Agilent) as described in ${ }^{37}$. Cells were infected $24 \mathrm{~h}$ before the start of the experiment to induce Nf2 gene expression. An empty adenoviral vector was used as a control (EV). shRNAexpressing lentiviruses were generated by co-transfecting 293T cells with pLKO-puro.1 vectors and the packaging vectors $\triangle$ VPR and VSVG (FuGENE, Promega). Viruses were harvested 24$48 \mathrm{~h}$ post transfection. shScr or shEzrin expressing lentiviruses were stably expressed in LDCs and selected in $4 \mu \mathrm{g} / \mathrm{ml}$ puromycin.

\section{Antibodies}

The following primary antibodies were used: anti-ezrin mouse monoclonal antibody (mAb) (1:500; MA5-13862, Invitrogen); anti-N-cadherin mouse mAb (IF 1:500, WB 1:1000; clone 32, BD Biosciences); anti- $\beta$-catenin mouse mAb (1:500; clone 14, BD Biosciences); anti-E-cadherin mouse mAb (WB 1:1000; clone 36, BD Biosciences); anti-pAkt (S473) rabbit mAb (IF 1:100, WB 1:1000; D9E, Cell Signaling Technology); anti-Akt rabbit pAb (1:1000; 9272, Cell Signaling Technology); anti-pERK1/2 (Thr202/Tyr204) rabbit mAb (1:1000, D13.14.4E, Cell Signaling Technology); anti-ERK1/2 rabbit mAb (1:1000, 137F5, Cell Signaling Technology); anti-pEGFR (Tyr1068) rabbit pAb (1:1000, 2234, Cell Signaling Technology); anti-EGFR rabbit mAb (IF 1:100, WB 1:1000; D38B1, Cell Signaling Technology), anti-ErbB2 rabbit pAb (1:100; A0485, Agilent), anti-pErbB3 (Tyr1289) rabbit mAb (1:500; 21D3, Cell Signaling Technology) anti-ErbB3 mouse mAb (1:100; RTJ2, Invitrogen); anti-pS6 (S235/236) rabbit pAb (1:100; 2211, Cell Signaling Technology); anti-Nrg1 rabbit pAb (1:100; ab191139, Abcam); anti-TGF $\alpha$ rabbit pAb (1:100; ab9585, Abcam); anti-integrin alpha 6 rabbit mAb (1:1000; ab181551, Abcam); antilaminin rabbit pAb (1:1000; ab11575, Abcam); anti-actin mouse mAb (1:1000; A4700, Sigma); anti-tubulin rabbit pAb (1:1000, T-3559, Sigma). Alexa Fluor 647-phalloidin or Rhodaminephalloidin (1:500; Thermo-Fisher) was used to label F-actin. Species-specific secondary 
antibodies conjugated to Alexa Fluor 488, 555, or 647 (Invitrogen) were used for IF. DAPI (Invitrogen) was used to label nuclei.

\section{Time lapse imaging}

60,000 cells per condition were plated and allowed to adhere for 15 min before washing away unbound cells. Images of regions of interest were captured at 20x magnification at time intervals of 5 min between cycles for $24 \mathrm{~h}$. Cells were imaged on a fully automated Nikon TiE microscope (Micro Device Instruments) equipped with a biochamber heated at $37^{\circ} \mathrm{C}$ and $5 \% \mathrm{CO}_{2}$ as described in [REF 72]. ${ }^{71}$

\section{Immunofluorescence microscopy}

Cells for immunofluorescence were plated on glass coverslips coated with poly-L-lysine and laminin $24-48 \mathrm{~h}$ before staining. Cells were fixed in 4\% paraformaldehyde in PBS for $15 \mathrm{~min}$ at room temperature, then permeabilized in $0.2 \%$ Triton $\mathrm{X}-100$ for $10 \mathrm{~min}$. Primary and secondary antibodies were diluted in PBS with 1\% BSA and incubated for $1 \mathrm{~h}$ at room temperature. Alexa Fluor-Phalloidin was added with secondary antibodies. Coverslips were mounted with Prolong Gold Antifade Mountant (Thermo Fisher). Cells were imaged with an inverted laser scanning confocal microscope (LSM710; Carl Zeiss) equipped with a 63x oil immersion objective (Plan Apochromat NA 1.4; Carl Zeiss). DAPI was excited with a 405-nm laser line of a diode laser. Alexa Fluor 488, Alexa Fluor 555, and Rhodamine probes were excited with the 488-nm or 514$\mathrm{nm}$ laser line of an argon laser. Texas-Red fluorescent probes were excited with the 561-nm laser line of a helium-neon laser. Alexa Fluor 647 probes were excited with the 633-nm laser line of a helium-neo laser. Images were acquired as single images or zstacks in sequential mode using ZEN Black software (2012; Carl Zeiss).

\section{Western blotting}


Cells were lysed in RIPA buffer (50 mM Tris, $\mathrm{pH} 7.4,1 \%$ Triton X-100, $1 \%$ SDS, $0.5 \%$ sodium deoxycholate, $150 \mathrm{mM} \mathrm{NaCl}, 1 \mathrm{mM}$ EDTA, $1 \mathrm{mM}$ EGTA, and protease inhibitors). Cell debris was cleared by centrifugation $\left(18,000 \mathrm{~g}\right.$ for $10 \mathrm{~min}$ at $\left.4^{\circ} \mathrm{C}\right)$ and equal amounts of protein were separated by SDS-PAGE, transferred to PVDF membranes, and immunoblotted with primary antibody overnight at $4^{\circ} \mathrm{C}$ in $5 \%$ milk or $5 \%$ BSA (phospho-specific antibody).

\section{Macropinocytosis Assay}

For in vitro assay, cells were plated on poly-L-lysine and laminin coated glass coverslips and incubated for 24-48 h until close to confluent. Cells were starved of serum and/or Nrg overnight in either complete growth medium or glutamine free medium. Dextran-488, (Oregon Green 488; $70,000 \mathrm{da}$, anionic, lysine fixable) or was added to cells along with growth factor when appropriate $(0.5 \mathrm{mg} / \mathrm{ml})$ and incubated for 30 minutes at $37^{\circ} \mathrm{C}$. Cells were rinsed 3 times with cold PBS and fixed with $4 \%$ paraformaldehyde. For the in vivo/ex vivo assay, PostnCre;Nf $2^{\text {flox/flox }}$ mice were injected with 250mg/kg FITC-Ficoll (70,000 Da, Millipore Sigma 51731$1 G)$ and dorsal root ganglia (DRG) were dissected $24 \mathrm{~h}$ later. Dissected tumors were incubated overnight in N2 growth medium without $\mathrm{Nrg}$ and then with $1 \mathrm{mg} / \mathrm{mL}$ dextran-TMR (10,000 Da, Molecular Probes D1817) for $1 \mathrm{~h}$ in complete N2 growth medium with Nrg. For the EIPA ex vivo assay, DRGs were dissected from Postn-Cre;Nf ${ }^{\text {floxfflox }}$ mice, incubated $24 \mathrm{~h}$ in N2 growth medium without Nrg with DMSO or EIPA $(500 \mu \mathrm{M})$ pretreatment. DRGs were then incubated for $30 \mathrm{~min}$ in N2 growth medium with $\mathrm{Nrg}$ and $1 \mathrm{mg} / \mathrm{mL}$ dextran-TMR. After treatments, DRGs were directly frozen in OCT blocks and sectioned at $8 \mu \mathrm{m}$.

\section{EV production and uptake}

EVs were harvested as previously described (Reategui et al. 2018) from the conditioned medium of $\sim 1.0 \times 10^{8}$ EO771-LMB cells expressing tandem dimer Tomato (tdTomato) fused to the NH2-terminus to a palmitoylation signal (Palm-tdTomato)(kindly provided by Drs. Xandra 
Breakfield and Shannon Stott). Medium was collected and centrifuged at $300 \mathrm{~g}$ for $10 \mathrm{~min}$ and at $2000 \mathrm{~g}$ for $10 \mathrm{~min}$. Supernatants were filtered (0.8 $\mu \mathrm{m}$; EMD Millipore) and ultracentrifuged at $100,000 \mathrm{~g}$ for $90 \mathrm{~min}$ at $4^{\circ} \mathrm{C}$ (Optima L-90K Ultracentrifuge, Beckman Coulter). Pelleted EVs were resuspended in $200 \mu$ I PBS. EVs were added to cells grown on poly-L-lysine and laminin coated glass coverslips at $\sim 10^{6}$ per $0.7 \mathrm{~cm}^{2}$ and incubated for $4 \mathrm{~h}$ at $37^{\circ} \mathrm{C}$. Cells were then fixed and stained with DAPI.

\section{Nutrient deprivation and conditioned medium experiments}

Cells were plated in complete culture medium and allowed to grow for $24-48 \mathrm{~h}$ until they reached near confluence. Complete culture medium was replaced with glutamine-free or Nrg1free medium, as indicated and incubated for $24 \mathrm{~h}$. For experiments in schwannoma cells serum was also removed from the medium. For glutamate rescue experiments, $10 \mathrm{mM}$ L-glutamic acid (Millipore Sigma) was added to glutamine-free medium and incubated with cells for $24 \mathrm{~h}$. For conditioned medium experiments, medium was harvested from $\mathrm{Nf}^{-/-} \mathrm{SCs}$ grown on $60 \mathrm{~mm}$ plates in complete growth medium, glutamine-free medium, or Nrg1-free medium for $72 \mathrm{~h}$. Medium was centrifuged at $400 \mathrm{~g}$ for $5 \mathrm{~min}$ and filtered through a $0.45 \mu \mathrm{m}$ filter. $100 \mu \mathrm{l}$ of conditioned medium was added to each coverslip containing $\mathrm{Nf}^{-/-} \mathrm{SCs}$ for the indicated time.

\section{Quantitative RT-PCR}

Total cellular RNA was extracted using TRIzol (Thermo Fisher) and reverse transcribed with MMLV-RT (Promega) using oligo-dT primers. Fast Start Universal SYBR Green mix (Millipore Sigma) was used to amplify $0.5 \mu \mathrm{l}$ of the RT reaction in a $25 \mu$ l total reaction volume. Triplicate samples were run on a Light Cycler 480 system (Roche Applied Science) with cycling conditions of denaturation for 15 seconds at $95^{\circ} \mathrm{C}$, annealing for 1 minute at $60^{\circ} \mathrm{C}$, and extension at $60^{\circ}$ C, 45 cycles. Expression of GAPDH was used as an internal reference gene. 
Primer sequences are as follows: Gapdh forward (5'-AGGTCGGTGTGAACGGATTTG-3'), reverse (5'-TGTAGACCATGTAGTTGAGGTCA-3'); Nrg1 forward (5'-

TCATCTTCTAGCGAGATGTCTG-3'), reverse (5'-CAGACATCTCGCTAGAAGATGA-3'); Nrg1, Typel forward (5'- GGGAAGGGCAAGAAGAAGG-3'), reverse (5'-

TTTCACACCGAAGCACGAGC-3'); Nrg1, Typell/ forward (5'-

ACTCAGCCACAAACAACAGAAAC 3'), reverse (5'-GAAGCACTCGCCTCCATT-3'); Tgfa forward (5'-CACTCTGGGTACGTGGGTG-3'), reverse (5'-CACAGGTGATAATGAGGACAGC3'); Egf forward (5'-AGCATCTCTCGGATTGACCCA-3'), reverse (5'CCTGTCCCGTTAAGGAAAACTCT-3'); Lama2 forward (5'-TCCCAAGCGCATCAACAGAG-3'), reverse (5'-CAGTACATCTCGGGTCCTTTTTC-3'); Itga6 forward (5'TGCAGAGGGCGAACAGAAC-3'), reverse (5'-CGTGCTGCCGTTTCTCATATC-3'); Dag1 forward (5'- CAGACGGTACGGCTGTTGTC-3', reverse (5'-AGTGTAGCCAAGACGGTAAGG3'); and Egfr forward (5'-GCCATCTGGGCCAAAGATACC-3'), reverse (5'-

GTCTTCGCATGAATAGGCCAAT-3'); Cdh2 forward (5'-CTCCAACGGGCATCTTCATTAT-3', reverse (5'- CAAGTGAAACCGGGCTATCAG-3'); Sox2 forward (5'-

AACGTTCTAGATTGTACTAAA-3', reverse (5'- AACAGTTACGTTTCCAACTTA-3').

\section{Immunohistochemistry and tissue staining}

Immunofluorescence staining was performed on frozen sections dissected from Postn-

Cre;Nf $2^{\text {floxfllox }}$ mice using a standard protocol: after air drying the frozen sections, they were fixed in formaldehyde $3.7 \%$ for 15 min, washed in PBS 3 times, incubated in a blocking/permeabilization buffer (PBS+5\%NGS+0.3\%Triton) for $1 \mathrm{~h}$. Sections were then incubated with primary antibodies overnight at $4^{\circ} \mathrm{C}$, washed with PBS 3 times and incubated with secondary antibodies for $1 \mathrm{~h}$ at room temperature. Nuclear staining was performed with Hoechst $33258(0.2 \mu \mathrm{g} / \mu \mathrm{L})$ and sections were mounted with Fluorescence Mounting Medium (Dako S3023). For staining of macropinocytosis assays, slides were washed quickly in PBS 
before fixation to reduce nonspecific background and then incubated with Hoechst before coverslip mounting $^{72}$. Images were acquired as single-plan in sequential mode using a SP8 Light-Sheet confocal microscope and the LAS X software (Leica). Standard protocols were used for immunofluorescent staining of formalin fixed paraffin embedded tissue. Briefly, tissues were dewaxed in xylene and rehydrated, followed by antigen retrieval in citrate buffer by incubation for $20 \mathrm{~min}$ at $95^{\circ} \mathrm{C}$. Sections were then blocked in $5 \%$ goat serum, $1 \%$ BSA in TBST for $1 \mathrm{~h}$ and then incubated with primary antibodies overnight at $4^{\circ} \mathrm{C}$. Sections were then washed, incubated with secondary antibodies for $1 \mathrm{~h}$ at room temperature, and incubated with DAPI to label nuclei prior to mounting on glass slides with Prolong Gold mounting media. Slides were scanned on a Vectra 3 whole slide scanning micrcoscope (Akoya Biosciences), viewed using Phenochart software, and spectrally unmixed using InForm software.

\section{Image analysis and statistics}

ImageJ software (version 2.0, National Institutes of Health) was used for all image processing and analysis. The displayed images were produced from single confocal slices or maximum projections of zstack images. Background was removed with rolling ball background subtraction. Lookup tables were applied to produce final images. Ruffle area was determined by measuring the ratio of cortical F-actin area over cell area $\left(\mu \mathrm{m}^{2}\right)$ in threshold images of maximum intensity projections (MIP) of 3D zstacks. Fluorescence intensity was measured by applying a threshold mask to a region of interest $(\mathrm{ROI})$ and calculating the area of positive signal to the total surface area of the cell, field of view, or region of interest. For cell polarity measurements, cell poles were either counted by hand in time lapse images or the aspect ratio was calculated using the shape descriptor measurement tool in ImageJ. Dextran or EV uptake was measured using the analyze particles function to determine the ratio of total particle area to total cell area in threshold images. For quantification of the ex vivo macropinocytosis inhibition by EIPA, analysis was performed with 5 single-plane confocal images per conditions, processed as described ${ }^{72}$. 
Whole slide scans of DRG lesions from Postn-Cre;Nf2 flox/flox $^{\text {mice }}$ were imported into HALO ${ }^{\text {TM }}$ digital image analysis software version 3.2 (Indica Labs). We then developed a digital image analysis algorithm using the $\mathrm{HALO}{ }^{\mathrm{TM}}$ multiplex $\mathrm{IHC}$ algorithm to achieve single cell segmentation of nucleus and cytoplasm and detect Nrg1 and TGF $\alpha$ at a single cell resolution in DRG lesions. Cell recognition and nuclear segmentation was trained and optimized in three randomly selected regions from each section. Thresholds for positive staining for Nrg1 and TGF $\alpha$ were set using 3 randomly selected regions close to and far from DRG soma. A supervised machine learning algorithm (Random Forest classifier) was trained on three randomly selected regions to recognize tumor cells and exclude DRG soma. For spatial analysis, the HALO ${ }^{\mathrm{TM}}$ proximity analysis module was used to measure the percentage of Nrg1 positive and Nrg1 negative cells within a $25 \mu \mathrm{M}$ radius of DRG soma identified using the classifier algorithm. The Random Forest classifer was trained and optimized to identify TGF $\alpha$ positive clusters in DRG lesions and measure the mean cluster size of TGF $\alpha$ in 6 month and 12 month Postn-Cre;Nf $2^{\text {floxfllox }}$ mice. Data from all analyses was imported into Prism 8 for plotting graphs and statistical analysis. The unpaired two tailed Student's t test was used to compare groups.

Acknowledgments. We thank past and present members of the McClatchey lab for valuable discussions, particularly Christian Davidson for intellectual input in the early stages of this project; Berent Aldikacti for EO771-LMB cells and expertise in EV isolation; João Paulo OliveiraCosta for advice on HALO-based image analysis; Daniel Irimia and Xiao Wang for assistance with time lapse imaging; MGH Cancer Center/Molecular Pathology Confocal Core for access to confocal microscopy equipment. This work was supported by Department of Defense grants W81XWH1910156 and W81XWH2110446 (A.I.M, M.G.), a Drug Discovery Initiative Award from 
the Children's Tumor Foundation (A.I.M.), and an MGH American Cancer Society Institutional Research Grant (C.C.M.).

Author Contributions: The study was conceived and designed by C.C.M., J.V. M.G., and A.I.M. In vitro experiments were carried out by C.C.M. and C.H.L., In vivo experiments were carried out by J.V. Whole slide scanning and HALO image analysis were carried out by C.C.M, E.A. F., and S.L.S. Data were analyzed and interpreted by C.C.M, C.H.L., J.V., M.G., and A.I.M. The project was supervised by A.I.M and M.G. Drafting of the manuscript and preparation of figures was completed by C.C.M and A.I.M. All authors read and commented on the manuscript. 


\section{References}

1 Evans, D. G. Neurofibromatosis type 2 (NF2): a clinical and molecular review. Orphanet J Rare Dis 4, 16, doi:10.1186/1750-1172-4-16 (2009).

$2 \quad$ Plotkin, S. R. \& Wick, A. Neurofibromatosis and Schwannomatosis. Semin Neurol 38, 7385, doi:10.1055/s-0038-1627471 (2018).

3 Jordan, J. T. \& Plotkin, S. R. Benign Intracranial Tumors. Neurol Clin 36, 501-516, doi:10.1016/j.ncl.2018.04.007 (2018).

$4 \quad$ Ardern-Holmes, S., Fisher, G. \& North, K. Neurofibromatosis Type 2. J Child Neurol 32, 922, doi:10.1177/0883073816666736 (2017).

5 Agnihotri, S. et al. The genomic landscape of schwannoma. Nat Genet 48, 1339-1348, doi:10.1038/ng.3688 (2016).

6 Plotkin, S. R. et al. Audiologic and radiographic response of NF2-related vestibular schwannoma to erlotinib therapy. Nat Clin Pract Oncol 5, 487-491, doi:10.1038/ncponc1157 (2008).

7 Karajannis, M. A. et al. Phase II trial of lapatinib in adult and pediatric patients with neurofibromatosis type 2 and progressive vestibular schwannomas. Neuro Oncol 14, 1163-1170, doi:10.1093/neuonc/nos146 (2012).

8 Goutagny, S. et al. Phase II study of mTORC1 inhibition by everolimus in neurofibromatosis type 2 patients with growing vestibular schwannomas. J Neurooncol 122, 313-320, doi:10.1007/s11060-014-1710-0 (2015).

9 Erlandson, R. A. \& Woodruff, J. M. Peripheral nerve sheath tumors: an electron microscopic study of 43 cases. Cancer 49, 273-287, doi:10.1002/10970142(19820115)49:2<273::aid-cncr2820490213>3.0.co;2-r (1982).

10 Waggener, J. D. Ultrastructure of benign peripheral nerve sheath tumors. Cancer 19, 699-709, doi:10.1002/1097-0142(196605)19:5<699::aid-cncr2820190516>3.0.co;2-h (1966).

11 Wippold, F. J., 2nd, Lubner, M., Perrin, R. J., Lammle, M. \& Perry, A. Neuropathology for the neuroradiologist: Antoni A and Antoni B tissue patterns. AJNR Am J Neuroradiol 28, 1633-1638, doi:10.3174/ajnr.A0682 (2007).

12 Lewis, D. et al. The microenvironment in sporadic and neurofibromatosis type Il-related vestibular schwannoma: the same tumor or different? A comparative imaging and neuropathology study. J Neurosurg, 1-11, doi:10.3171/2020.3.JNS193230 (2020).

13 Stierli, S., Imperatore, V. \& Lloyd, A. C. Schwann cell plasticity-roles in tissue homeostasis, regeneration, and disease. Glia 67, 2203-2215, doi:10.1002/glia.23643 (2019).

14 Heller, B. A. et al. Functionally distinct PI 3-kinase pathways regulate myelination in the peripheral nervous system. J Cell Biol 204, 1219-1236, doi:10.1083/jcb.201307057 (2014).

15 Wilson, E. R., Della-Flora Nunes, G., Weaver, M. R., Frick, L. R. \& Feltri, M. L. Schwann cell interactions during the development of the peripheral nervous system. Dev Neurobiol, doi:10.1002/dneu.22744 (2020). 
16 Lewallen, K. A. et al. Assessing the role of the cadherin/catenin complex at the Schwann cell-axon interface and in the initiation of myelination. J Neurosci 31, 3032-3043, doi:10.1523/JNEUROSCI.4345-10.2011 (2011).

17 Chan, J. R. et al. The polarity protein Par-3 directly interacts with p75NTR to regulate myelination. Science 314, 832-836, doi:10.1126/science.1134069 (2006).

18 Poliak, S., Matlis, S., Ullmer, C., Scherer, S. S. \& Peles, E. Distinct claudins and associated PDZ proteins form different autotypic tight junctions in myelinating Schwann cells. $J$ Cell Biol 159, 361-372, doi:10.1083/jcb.200207050 (2002).

19 Ozcelik, M. et al. Pals1 is a major regulator of the epithelial-like polarization and the extension of the myelin sheath in peripheral nerves. J Neurosci 30, 4120-4131, doi:10.1523/JNEUROSCI.5185-09.2010 (2010).

20 Boucanova, F. \& Chrast, R. Metabolic Interaction Between Schwann Cells and Axons Under Physiological and Disease Conditions. Front Cell Neurosci 14, 148, doi:10.3389/fncel.2020.00148 (2020).

21 Salzer, J. L. Schwann cell myelination. Cold Spring Harb Perspect Biol 7, a020529, doi:10.1101/cshperspect.a020529 (2015).

22 James, M. F. et al. Regulation of mTOR complex 2 signaling in neurofibromatosis 2deficient target cell types. Mol Cancer Res 10, 649-659, doi:10.1158/1541-7786.MCR-110425-T (2012).

23 Garcia-Martinez, J. M. \& Alessi, D. R. mTOR complex 2 (mTORC2) controls hydrophobic motif phosphorylation and activation of serum- and glucocorticoid-induced protein kinase 1 (SGK1). Biochem J 416, 375-385, doi:10.1042/BJ20081668 (2008).

24 Fehon, R. G., McClatchey, A. I. \& Bretscher, A. Organizing the cell cortex: the role of ERM proteins. Nat Rev Mol Cell Biol 11, 276-287, doi:10.1038/nrm2866 (2010).

25 Boggiano, J. C. \& Fehon, R. G. Growth control by committee: intercellular junctions, cell polarity, and the cytoskeleton regulate Hippo signaling. Dev Cell 22, 695-702, doi:10.1016/j.devcel.2012.03.013 (2012).

26 McClatchey, A. I. \& Fehon, R. G. Merlin and the ERM proteins--regulators of receptor distribution and signaling at the cell cortex. Trends Cell Biol 19, 198-206, doi:10.1016/j.tcb.2009.02.006 (2009).

27 Hebert, A. M., DuBoff, B., Casaletto, J. B., Gladden, A. B. \& McClatchey, A. I. Merlin/ERM proteins establish cortical asymmetry and centrosome position. Genes Dev 26, 27092723, doi:10.1101/gad.194027.112 (2012).

28 Gladden, A. B., Hebert, A. M., Schneeberger, E. E. \& McClatchey, A. I. The NF2 tumor suppressor, Merlin, regulates epidermal development through the establishment of a junctional polarity complex. Dev Cell 19, 727-739, doi:10.1016/j.devcel.2010.10.008 (2010).

29 Das, T. et al. A molecular mechanotransduction pathway regulates collective migration of epithelial cells. Nat Cell Biol 17, 276-287, doi:10.1038/ncb3115 (2015).

30 Yi, C. et al. A tight junction-associated Merlin-angiomotin complex mediates Merlin's regulation of mitogenic signaling and tumor suppressive functions. Cancer Cell 19, 527540, doi:10.1016/j.ccr.2011.02.017 (2011). 
31 D'Angelo, R. et al. Interaction of ezrin with the novel guanine nucleotide exchange factor PLEKHG6 promotes RhoG-dependent apical cytoskeleton rearrangements in epithelial cells. Mol Biol Cell 18, 4780-4793, doi:10.1091/mbc.E06-12-1144 (2007).

32 Morrison, H. et al. Merlin/neurofibromatosis type 2 suppresses growth by inhibiting the activation of Ras and Rac. Cancer Res 67, 520-527, doi:10.1158/0008-5472.CAN-06-1608 (2007).

$33 \mathrm{Wu}, \mathrm{N}$. et al. The Hippo signaling functions through the Notch signaling to regulate intrahepatic bile duct development in mammals. Lab Invest 97, 843-853, doi:10.1038/labinvest.2017.29 (2017).

34 James, M. F. et al. NF2/merlin is a novel negative regulator of mTOR complex 1 , and activation of mTORC1 is associated with meningioma and schwannoma growth. Mol Cell Biol 29, 4250-4261, doi:10.1128/MCB.01581-08 (2009).

35 Lopez-Lago, M. A., Okada, T., Murillo, M. M., Socci, N. \& Giancotti, F. G. Loss of the tumor suppressor gene NF2, encoding merlin, constitutively activates integrindependent mTORC1 signaling. Mol Cell Biol 29, 4235-4249, doi:10.1128/MCB.01578-08 (2009).

36 Hamaratoglu, F. et al. The tumour-suppressor genes NF2/Merlin and Expanded act through Hippo signalling to regulate cell proliferation and apoptosis. Nat Cell Biol 8, 2736, doi:10.1038/ncb1339 (2006).

37 Chiasson-MacKenzie, C. et al. NF2/Merlin mediates contact-dependent inhibition of EGFR mobility and internalization via cortical actomyosin. J Cell Biol 211, 391-405, doi:10.1083/jcb.201503081 (2015).

38 Chiasson-MacKenzie, C. et al. Merlin/ERM proteins regulate growth factor-induced macropinocytosis and receptor recycling by organizing the plasma membrane:cytoskeleton interface. Genes Dev 32, 1201-1214, doi:10.1101/gad.317354.118 (2018).

39 Curto, M., Cole, B. K., Lallemand, D., Liu, C. H. \& McClatchey, A. I. Contact-dependent inhibition of EGFR signaling by Nf2/Merlin. J Cell Biol 177, 893-903, doi:10.1083/jcb.200703010 (2007).

40 Cole, B. K., Curto, M., Chan, A. W. \& McClatchey, A. I. Localization to the cortical cytoskeleton is necessary for $\mathrm{Nf2/merlin-dependent} \mathrm{epidermal} \mathrm{growth} \mathrm{factor} \mathrm{receptor}$ silencing. Mol Cell Biol 28, 1274-1284, doi:10.1128/MCB.01139-07 (2008).

41 Maitra, S., Kulikauskas, R. M., Gavilan, H. \& Fehon, R. G. The tumor suppressors Merlin and Expanded function cooperatively to modulate receptor endocytosis and signaling. Curr Biol 16, 702-709, doi:10.1016/j.cub.2006.02.063 (2006).

42 Lallemand, D. et al. Merlin regulates transmembrane receptor accumulation and signaling at the plasma membrane in primary mouse Schwann cells and in human schwannomas. Oncogene 28, 854-865, doi:10.1038/onc.2008.427 (2009).

43 Schulz, A. et al. Neuronal merlin influences ERBB2 receptor expression on Schwann cells through neuregulin 1 type III signalling. Brain 137, 420-432, doi:10.1093/brain/awt327 (2014).

44 Bloomfield, G. \& Kay, R. R. Uses and abuses of macropinocytosis. J Cell Sci 129, 26972705, doi:10.1242/jcs.176149 (2016). 
45 Thaxton, C. et al. Schwannomin/merlin promotes Schwann cell elongation and influences myelin segment length. Mol Cell Neurosci 47, 1-9, doi:10.1016/j.mcn.2010.12.006 (2011).

46 Newbern, J. \& Birchmeier, C. Nrg1/ErbB signaling networks in Schwann cell development and myelination. Semin Cell Dev Biol 21, 922-928, doi:10.1016/j.semcdb.2010.08.008 (2010).

47 Melendez-Vasquez, C. V. et al. Nodes of Ranvier form in association with ezrin-radixinmoesin (ERM)-positive Schwann cell processes. Proc Natl Acad Sci U S A 98, 1235-1240, doi:10.1073/pnas.98.3.1235 (2001).

48 Wanner, I. B. et al. Role of N-cadherin in Schwann cell precursors of growing nerves. Glia 54, 439-459, doi:10.1002/glia.20390 (2006).

49 Gess, B. et al. Inhibition of N-cadherin and beta-catenin function reduces axon-induced Schwann cell proliferation. J Neurosci Res 86, 797-812, doi:10.1002/jnr.21528 (2008).

50 Parrinello, S. et al. EphB signaling directs peripheral nerve regeneration through Sox2dependent Schwann cell sorting. Cell 143, 145-155, doi:10.1016/j.cell.2010.08.039 (2010).

51 Carmona-Fontaine, C. et al. Contact inhibition of locomotion in vivo controls neural crest directional migration. Nature 456, 957-961, doi:10.1038/nature07441 (2008).

52 Gehlhausen, J. R. et al. A murine model of neurofibromatosis type 2 that accurately phenocopies human schwannoma formation. Hum Mol Genet 24, 1-8, doi:10.1093/hmg/ddu414 (2015).

53 Ha, K. D., Bidlingmaier, S. M. \& Liu, B. Macropinocytosis Exploitation by Cancers and Cancer Therapeutics. Front Physiol 7, 381, doi:10.3389/fphys.2016.00381 (2016).

54 Hall, J. et al. Delivery of Therapeutic Proteins via Extracellular Vesicles: Review and Potential Treatments for Parkinson's Disease, Glioma, and Schwannoma. Cell Mol Neurobiol 36, 417-427, doi:10.1007/s10571-015-0309-0 (2016).

55 Vader, P., Mol, E. A., Pasterkamp, G. \& Schiffelers, R. M. Extracellular vesicles for drug delivery. Adv Drug Deliv Rev 106, 148-156, doi:10.1016/j.addr.2016.02.006 (2016).

56 Armstrong, J. P., Holme, M. N. \& Stevens, M. M. Re-Engineering Extracellular Vesicles as Smart Nanoscale Therapeutics. ACS Nano 11, 69-83, doi:10.1021/acsnano.6b07607 (2017).

57 Lee, S. W. et al. EGFR-Pak Signaling Selectively Regulates Glutamine Deprivation-Induced Macropinocytosis. Dev Cell 50, 381-392 e385, doi:10.1016/j.devcel.2019.05.043 (2019).

58 Ammoun, S. et al. ErbB/HER receptor activation and preclinical efficacy of lapatinib in vestibular schwannoma. Neuro Oncol 12, 834-843, doi:10.1093/neuonc/noq012 (2010).

59 Boucanova, F. et al. Disrupted function of lactate transporter MCT1, but not MCT4, in Schwann cells affects the maintenance of motor end-plate innervation. Glia 69, 124-136, doi:10.1002/glia.23889 (2021).

60 Saitoh, F., Wakatsuki, S., Tokunaga, S., Fujieda, H. \& Araki, T. Glutamate signals through mGluR2 to control Schwann cell differentiation and proliferation. Sci Rep 6, 29856, doi:10.1038/srep29856 (2016).

61 Giovannini, M. et al. mTORC1 inhibition delays growth of neurofibromatosis type 2 schwannoma. Neuro Oncol 16, 493-504, doi:10.1093/neuonc/not242 (2014). 
62 Hannan, C. J. et al. The inflammatory microenvironment in vestibular schwannoma. Neurooncol Adv 2, vdaa023, doi:10.1093/noajnl/vdaa023 (2020).

63 Poitelon, Y. et al. YAP and TAZ control peripheral myelination and the expression of laminin receptors in Schwann cells. Nat Neurosci 19, 879-887, doi:10.1038/nn.4316 (2016).

64 Totaro, A., Panciera, T. \& Piccolo, S. YAP/TAZ upstream signals and downstream responses. Nat Cell Biol 20, 888-899, doi:10.1038/s41556-018-0142-z (2018).

65 Beauchamp, R. L. et al. mTOR kinase inhibition disrupts neuregulin 1-ERBB3 autocrine signaling and sensitizes NF2-deficient meningioma cellular models to IGF1R inhibition. J Biol Chem 296, 100157, doi:10.1074/jbc.RA120.014960 (2021).

66 Giovannini, M. et al. Conditional biallelic Nf2 mutation in the mouse promotes manifestations of human neurofibromatosis type 2. Genes Dev 14, 1617-1630 (2000).

67 Giovannini, M. et al. Schwann cell hyperplasia and tumors in transgenic mice expressing a naturally occurring mutant NF2 protein. Genes Dev 13, 978-986 (1999).

68 Lindsley, A. et al. Identification and characterization of a novel Schwann and outflow tract endocardial cushion lineage-restricted periostin enhancer. Dev Biol 307, 340-355, doi:10.1016/j.ydbio.2007.04.041 (2007).

69 Manent, J., Oguievetskaia, K., Bayer, J., Ratner, N. \& Giovannini, M. Magnetic cell sorting for enriching Schwann cells from adult mouse peripheral nerves. J Neurosci Methods 123, 167-173 (2003).

70 James, M. F. et al. Modeling NF2 with human arachnoidal and meningioma cell culture systems: NF2 silencing reflects the benign character of tumor growth. Neurobiol Dis 29, 278-292, doi:10.1016/j.nbd.2007.09.002 (2008).

71 Wang, X., Hossain, M., Bogoslowski, A., Kubes, P. \& Irimia, D. Chemotaxing neutrophils enter alternate branches at capillary bifurcations. Nat Commun 11, 2385, doi:10.1038/s41467-020-15476-6 (2020).

72 Commisso, C., Flinn, R. J. \& Bar-Sagi, D. Determining the macropinocytic index of cells through a quantitative image-based assay. Nat Protoc 9, 182-192, doi:10.1038/nprot.2014.004 (2014). 
a.

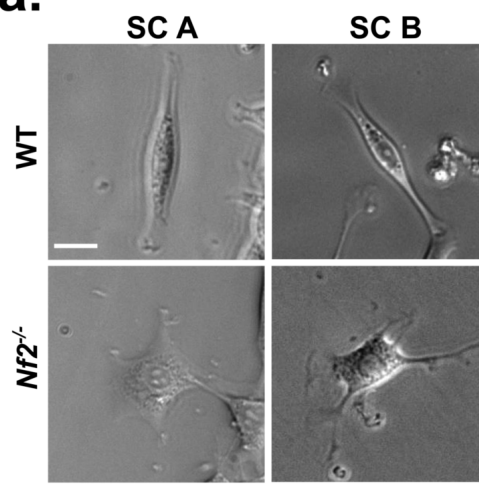

C.

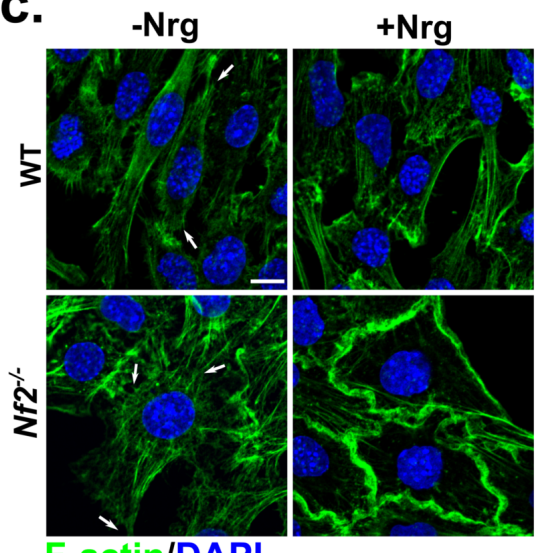

e.

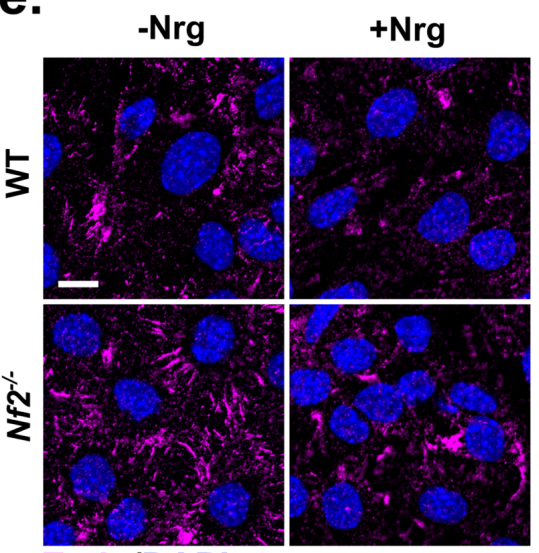

Ezrin/DAPI
$\operatorname{sc~C}$

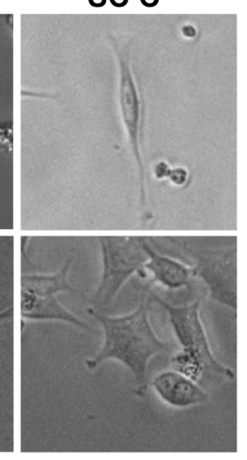

d.

b.

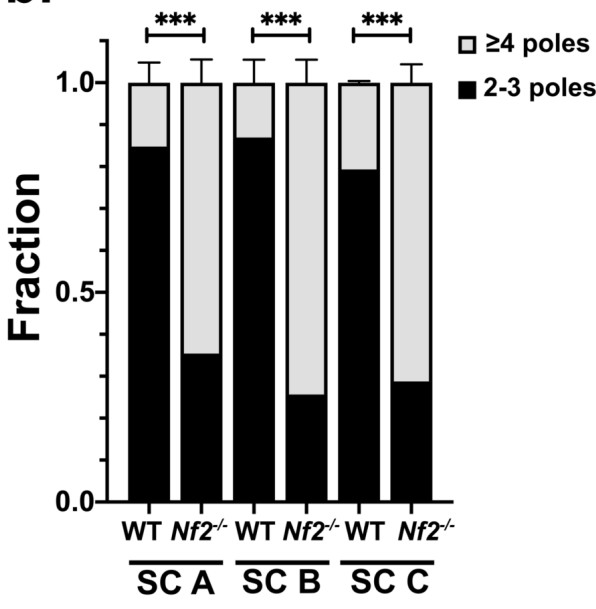

f.

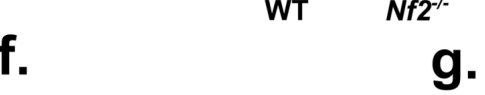

g.
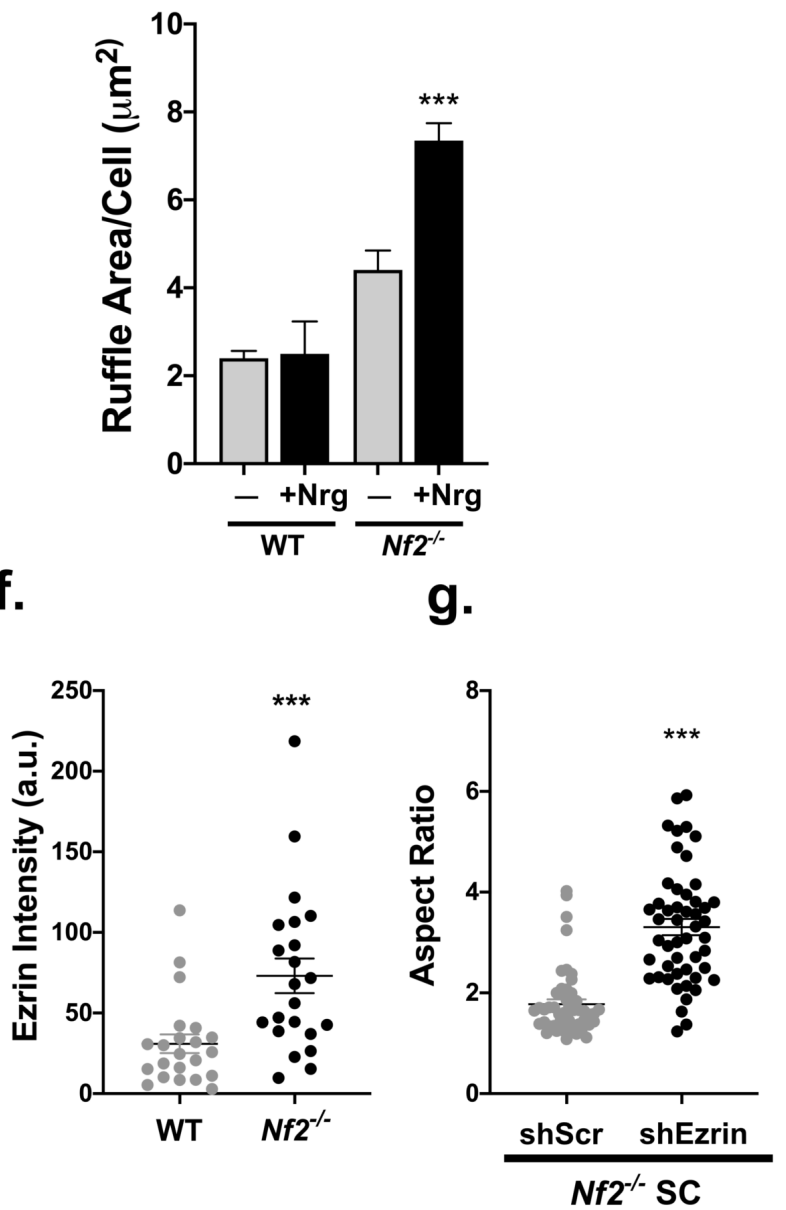
Figure 1. Nf2-deficient Schwann cells exhibit enhanced multipolarity and exaggerated cytoskeletal responses to the presence or absence of Nrg1. A. Representative phase contrast images of polarity in three independently generated $\mathrm{Nf}^{-/-}$and control parental $\mathrm{Nf} 2^{f l o x / f l o x}$ (WT) SC lines. B. Quantitation of multipolarity in three $N 2^{-/-}$and WT SC lines. Data are presented as the fraction of cells containing 2-3 poles or $\geq 4$ poles. Bars represent SEM for $N=3$ independent experiments with $n \geq 85$ cells per condition. C. Confocal images depicting F-actin (green) organization in early confluent WT and $\mathrm{Nf2}^{-/-}$SCs with and without stimulation with 10 $\mathrm{ng} / \mathrm{ml} \mathrm{Nrg1}$ for $10 \mathrm{~min}$. D. Quantitation of F-actin-containing ruffles in late confluent WT and Nf21- SCs with and without Nrg1 stimulation. Ruffle area was determined by measuring the ratio of cortical F-actin area over cell area $\left(\mu \mathrm{m}^{2}\right)$ in threshold images of MIP of 3D zstacks. Data are presented as mean + -- SEM of $n \geq 50$ cells per condition, $N=3$ independent experiments. $E$. Confocal images depicting ezrin (magenta) localization in WT and $\mathrm{Nf}^{-/-}$SCs with and without Nrg1 stimulation. F. Graph depicting Ezrin intensity in Nrg1 stimulated cortical ruffles in WT and $\mathrm{Nf}^{-/-}$SCs. Ezrin intensity was measured within ROls generated from threshold images of Factin. Lines represent mean $+/$ - SEM with $n=22$ cells per condition, $N=3$ independent experiments. G. Quantitation of polarity in $\mathrm{Nf}^{-/-} \mathrm{SC}$ infected with shSCR- or shEzrinexpressing lentiviruses; as determined by the aspect ratio (long to short axis). Lines represent mean $+/-$ SEM in $n=50$ cells per condition. $N=2$ independent experiments. $P$ values were calculated with unpaired two-tailed Student's t-test. ${ }^{* *} p<0.001$. Scale bars $=10 \mu \mathrm{m}$. 
a.

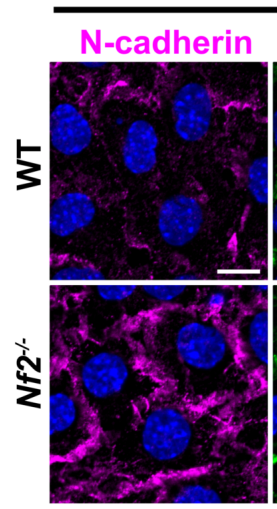

C.
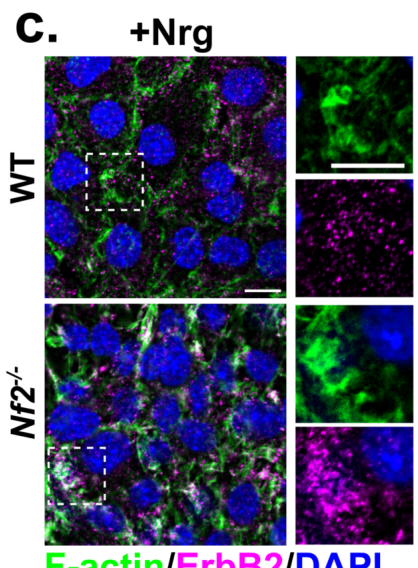

F-actin/ErbB2/DAPI

g.

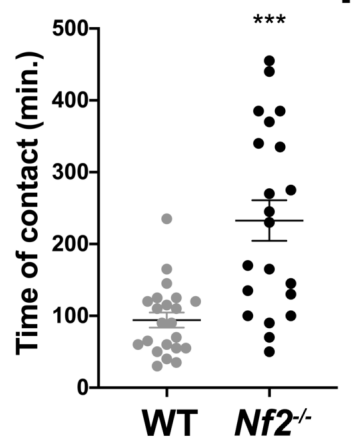

b.

Chiasson-MacKenzie_Figure2

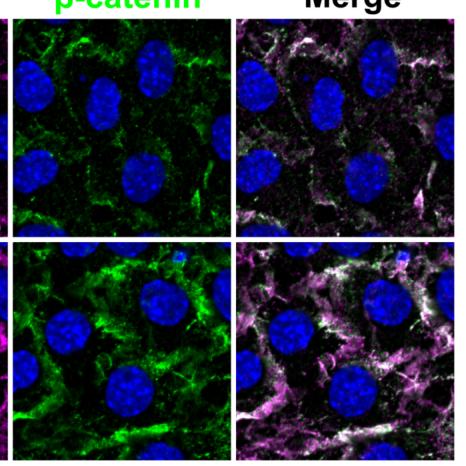

e.
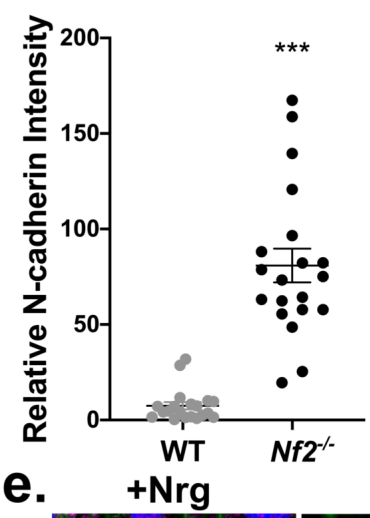

\begin{abstract}
d.
\end{abstract}

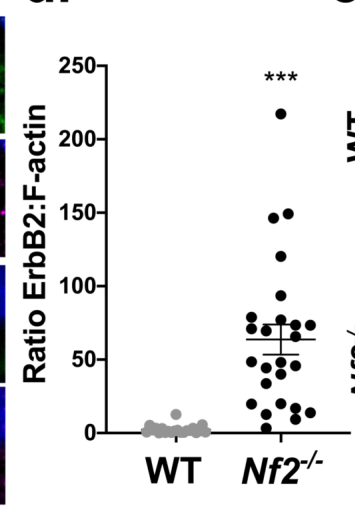

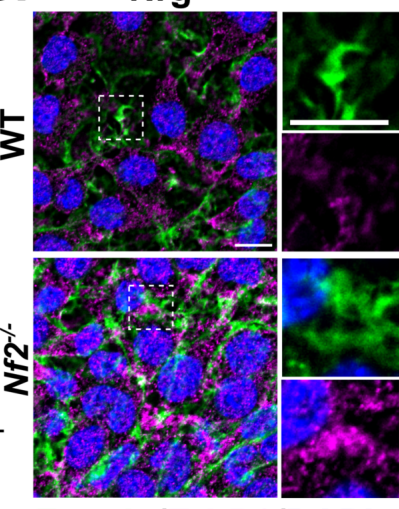

F-actin/ErbB3/DAPI

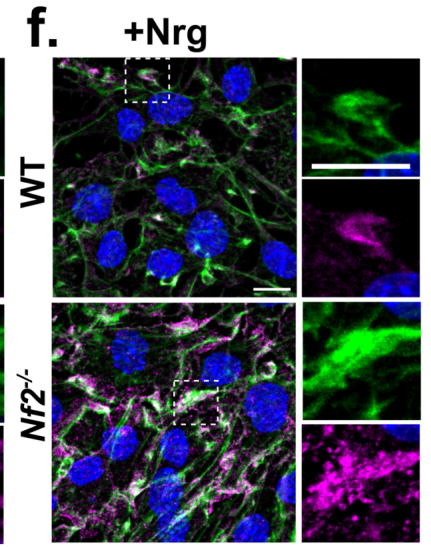

F-actin/pAkt/DAPI

i.

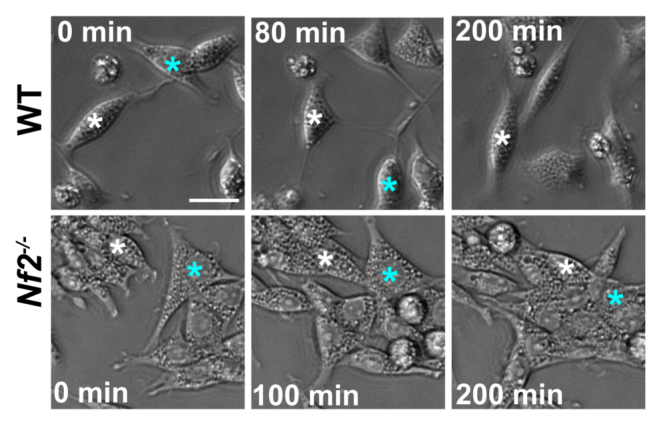

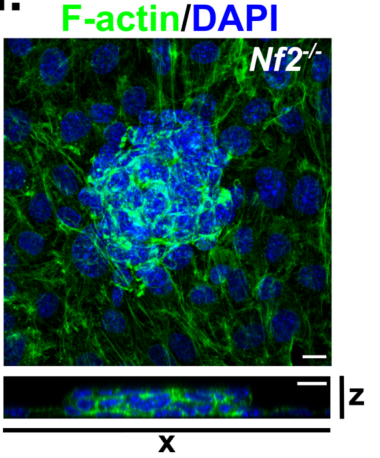


Figure 2. Adhesion and signaling in $\mathrm{Nf2}^{-/-} \mathrm{SCs}$ in the presence of $\mathrm{Nrg1}$. A. Confocal images depicting $\mathrm{N}$-cadherin (magenta) and $\beta$-catenin (green) localization in Nrg1 stimulated WT and $\mathrm{Nf2}^{-/-}$SCs. B. Quantitation of polarized N-cadherin intensity in Nrg1 stimulated WT and Nf2/SCs. Polarized N-cadherin intensity was measured by dividing the intensity of a ROI along the longest axis of a cell by the intensity of a ROI of equal size along the shortest axis of the cell. Graph represents mean +/- SEM in $n=20$ junctions per condition. N=3 independent experiments. C. Confocal images showing recruitment of ErbB2 (magenta) to Nrg1-induced F-actin (green) enriched cortical ruffles in WT and $\mathrm{Nf2}^{-/-}$SCs. D. Quantitation of the ratio of ErbB2 relative to Factin in Nrg1-induced ruffles in WT and $\mathrm{Nf}^{-/-}$SCs. Graph represents mean +/- SEM in n>20 cells per condition. $\mathrm{N}=3$ independent experiments. E. Nrg1-stimulated $\mathrm{Nf2}{ }^{-/-} \mathrm{SCs}$ labelled for ErbB3 (magenta) and F-actin (green) to show recruitment of ErbB3 to cortical ruffles. F. Confocal images showing activation of Akt (pAkt S473) in Nrg1-induced cortical ruffles in Nf2-SCs. G. Graph showing duration of sustained contact after WT and Nf2- SCs collide as calculated from time lapse phase contrast microscopy images. Lines represent mean +/- SEM in $\mathrm{n}>20$ cells per condition. $\mathrm{N}=3$ independent experiments. $\mathbf{H}$. Individual phase contrast images from time lapse microscopy showing the result of collision between WT or Nf2-- SCs. Asterisks mark two individual cells that come into contact. I. Top, MIP of late confluent $N f 2^{-/-}$SCs labelled for F-actin (green). Bottom, vertical x-z section of confocal zstack image showing tightly packed balls of cells that lose contact with the laminin-coated coverslip. P values were calculated with unpaired two-tailed Student's t-test. ${ }^{* *} \mathrm{p}<0.001$. Scale bars $=10 \mu \mathrm{m}$. See also Videos S1 and S2. 


\section{Chiasson-MacKenzie_Figure3}

a.

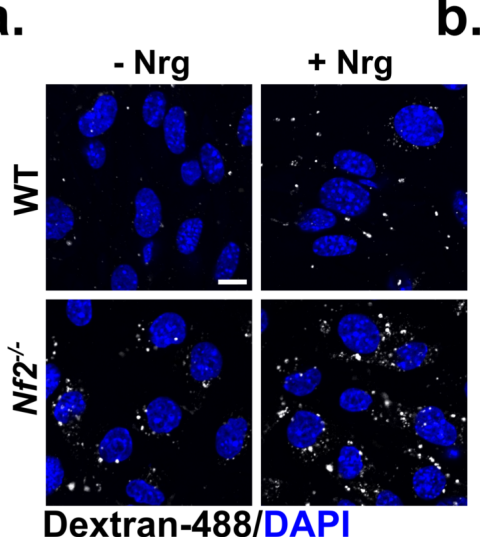

d.

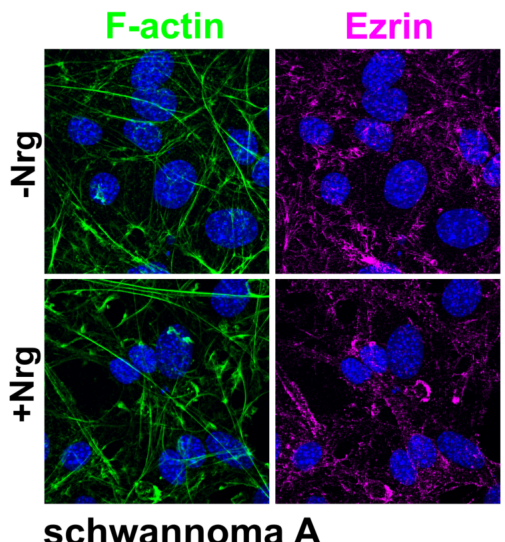

f.

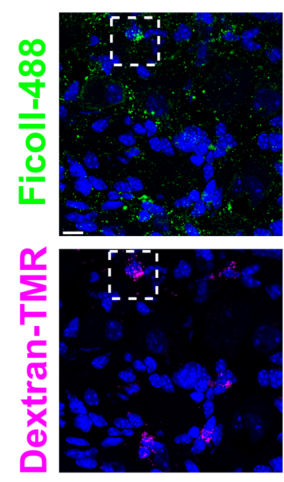

b.

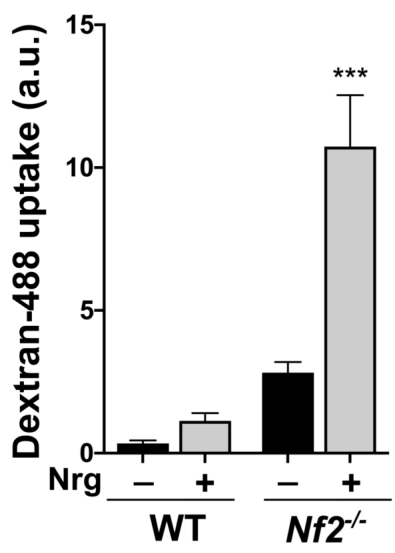

C.

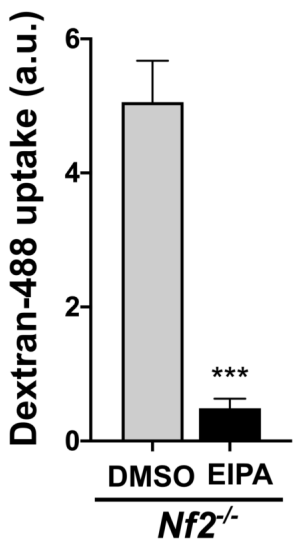

e.
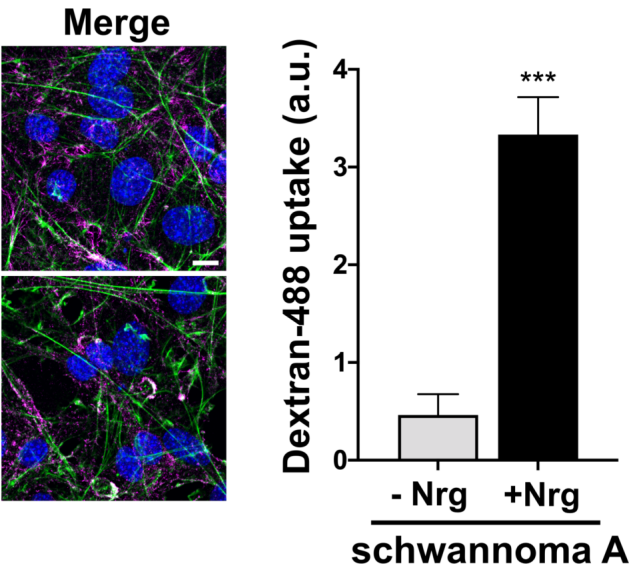

h.

Ex vivo

i. macropinocvtosis
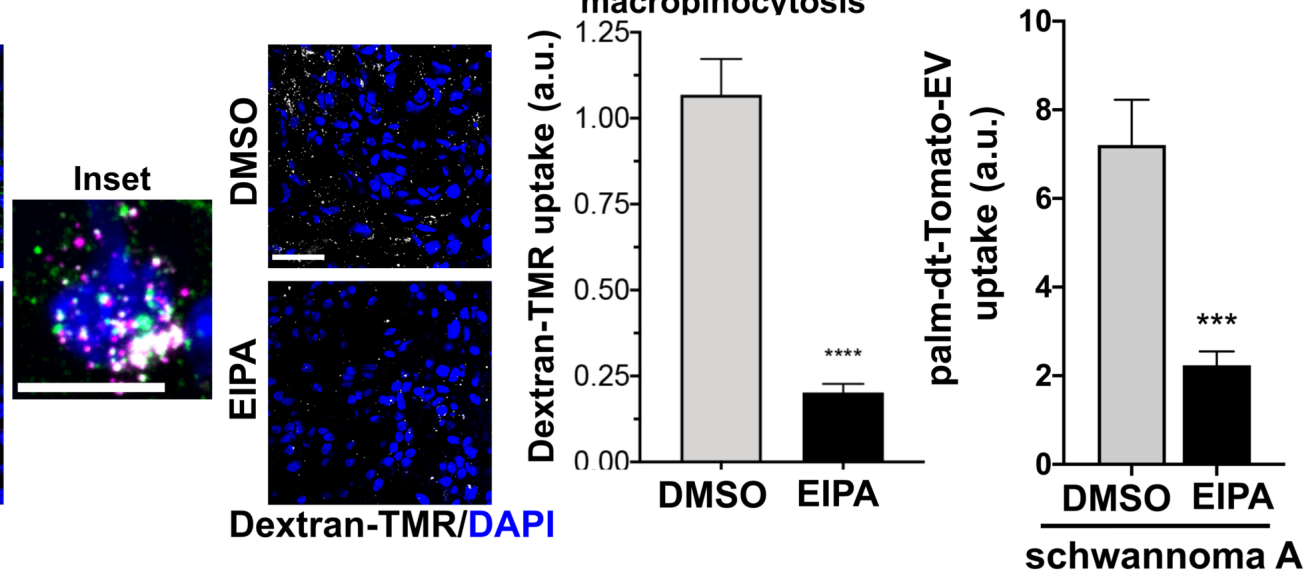
Figure 3: Nrg1-induced macropinocytosis and extracellular vesicle (EV) uptake in Nf2/-

SCs. A. Uptake of the macropinocytosis cargo dextran-488 with or without 30 min Nrg1stimulation in WT and Nf2-- SCs. B. Quantitation of dextran-488 uptake in WT and Nf2-- SCs with or without Nrg1-stimulation. Values represent mean $+/-$ SEM for $n=20$ cells per condition. $\mathrm{N}=3$ independent experiments. C. Graph showing dextran-488 uptake in Nf2-- SCs treated with vehicle (DMSO) or $50 \mu$ M EIPA. Bars represent mean $+/$ - SEM for $n=21$ cells per condition. $N=3$ independent experiments. D. Confocal images depicting F-actin (green) and ezrin (magenta) localization in $\mathrm{Nf}^{-/-}$mouse schwannoma cells with or without stimulation with Nrg1. E. Quantitation of dextran-488 uptake in $\mathrm{Nf2}^{-/-}$schwannoma cells with or without Nrg1 stimulation. Bars represent mean $+/$ - SEM for $n=20$ cells per condition. $\mathrm{N}=3$ independent experiments. $\mathbf{F}$. Confocal images of macropinocytic uptake of FITC-Ficoll (tail vein injected) and dextran-488 (applied ex vivo) in an early DRG lesion in a 12 month-old Postn-Cre;Nf2 floxflox mouse. Scale bar=10 $\mu \mathrm{m}$. G. Confocal images of dextran-TMR uptake with and without EIPA treatment (0.5 $\mathrm{mM}$ ) ex vivo in a DRG lesion in a Postn-Cre;Nf2 floxflox mouse. Scale bar=30 $\mu \mathrm{m}$. H. Graph measuring dextran-TMR uptake with and without EIPA treatment ex vivo in a DRG lesion. Bars represent mean +/- SEM, N=3. I. Uptake of Palm-tdTomato-labelled EVs into $\mathrm{Nf}^{-/-}$schwannoma cells after treatment with vehicle (DMSO) or $50 \mu \mathrm{M}$ EIPA. Bars represent mean +/- SEM for $n=40$ cells for DMSO, $n=18$ cells for EIPA. $N=3$ independent experiments. $P$ values were calculated with unpaired two-tailed Student's t-test. ${ }^{* * *} p<0.001$. Scale bars $=10 \mu \mathrm{m}$. See also Video S3. 

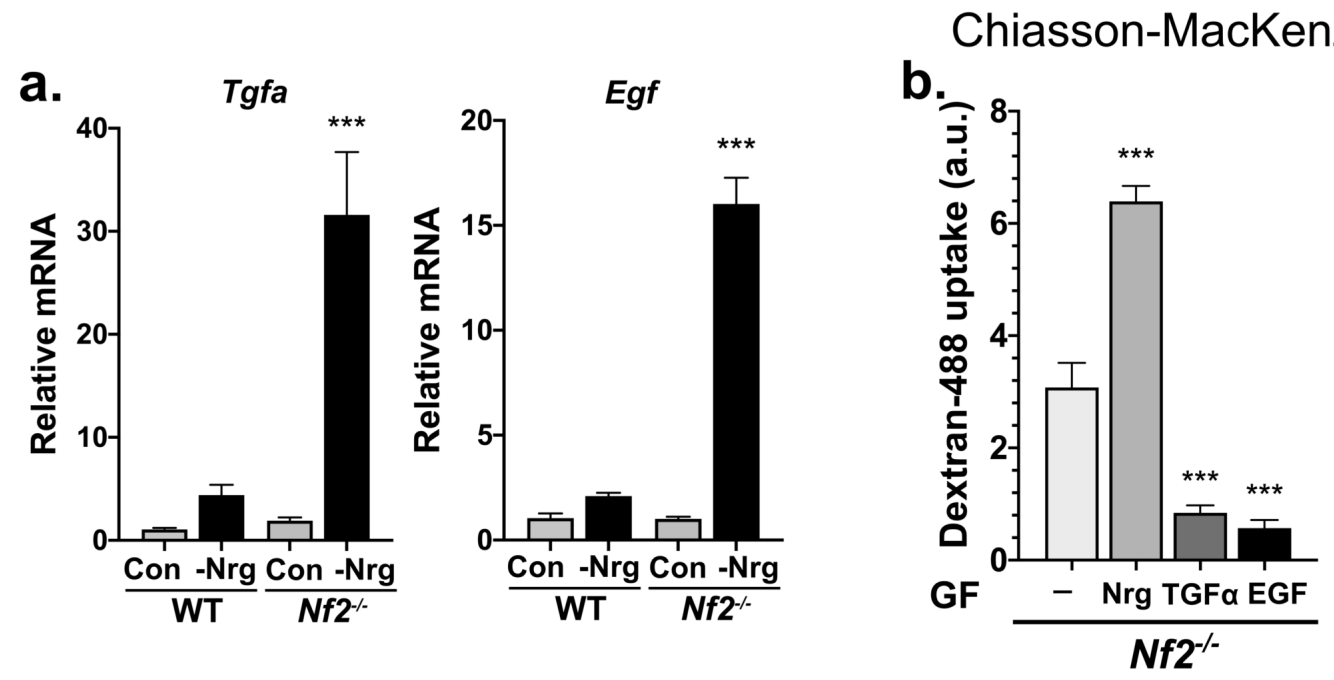

C.
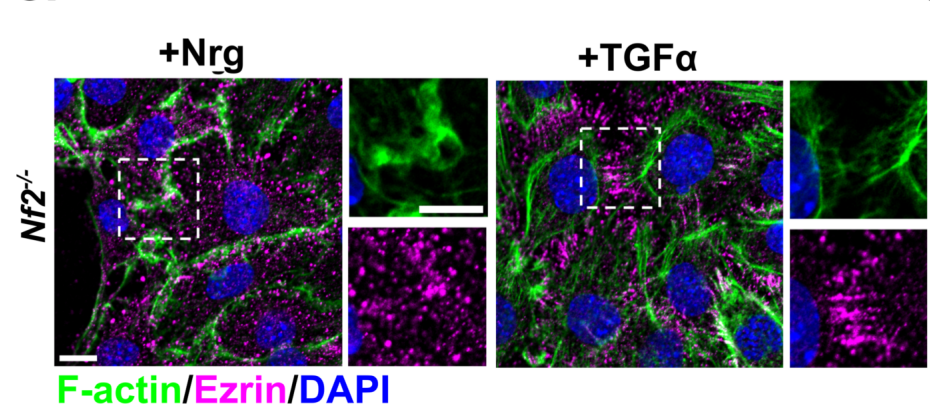

d.
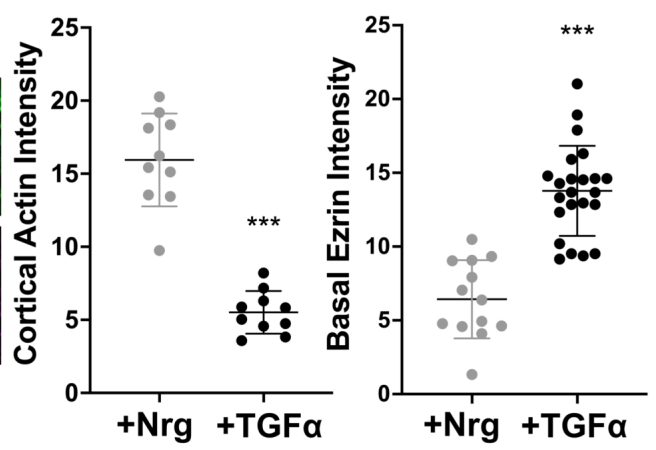

e.
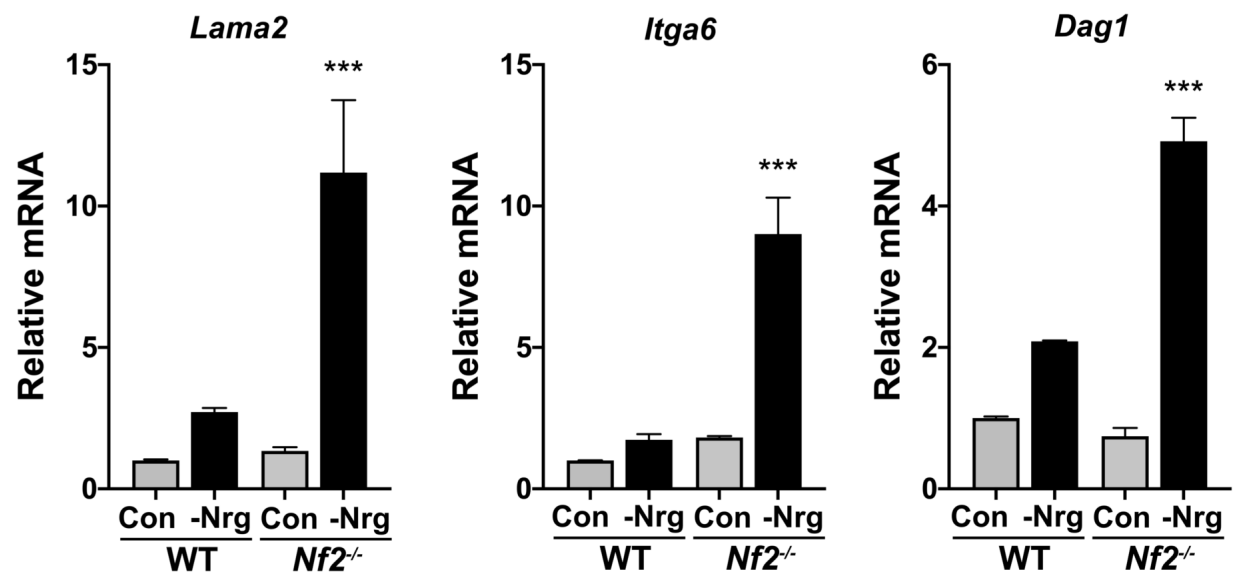
Figure 4. $\mathrm{Nf2}^{-/-} \mathrm{SCs}$ upregulate basal polarity components upon Nrg1-deprivation. A. mRNA expression of Tgfa and Egf in WT and $\mathrm{Nf}^{-/-} \mathrm{SCs}$ grown under steady-state conditions or deprived of Nrg1 for $24 \mathrm{~h}$. Data is presented as mean +/- SEM relative to WT mRNA levels, $\mathrm{N}=3$ independent experiments B. Quantitation of macropinocytic dextran-488 uptake in $\mathrm{Nf}^{-/-} \mathrm{SCs}$ starved of Nrg1 overnight and stimulated with $10 \mathrm{ng} / \mathrm{ml} \mathrm{Nrg} 1$, TGF $\alpha$, or EGF. C. Confocal images depicting the distribution of F-actin (green) and ezrin (magenta) in Nf2/- SCs starved of Nrg1 overnight and stimulated with $10 \mathrm{ng} / \mathrm{ml} \mathrm{Nrg1} \mathrm{or} \mathrm{TGF} \alpha$. D. Quantitation of cortical actin ruffles and basal ezrin intensity in cells in (C). Cortical actin or basal ezrin were measured in single plane threshold images from 3D zstacks as the ratio between actin or ezrin area and total cell area for $n \geq 20$ cells per condition. $N=3$ independent experiments. Lines represent mean $+/$ SEM. E. mRNA expression of Lam2, Itga6, and Dag1 in WT and Nf2-/- SCs under steady state conditions or after $24 \mathrm{~h} \mathrm{Nrg1-deprivation.} \mathrm{Data} \mathrm{is} \mathrm{presented} \mathrm{as} \mathrm{mean} \mathrm{+/-} \mathrm{SEM} \mathrm{relative} \mathrm{to} \mathrm{WT}$ mRNA levels, $N=3$ independent experiments. $P$ values were calculated with unpaired two-tailed Student's t-test. ${ }^{* * *} p<0.001$. Scale bars $=10 \mu \mathrm{m}$. 
a.

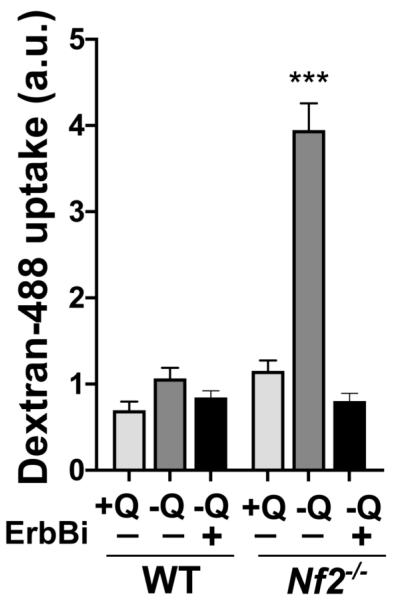

b.

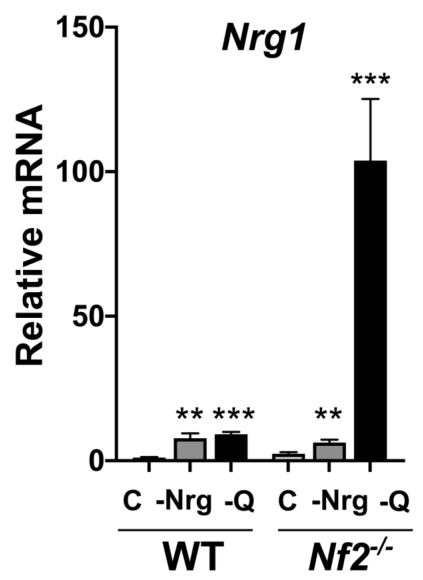

C.

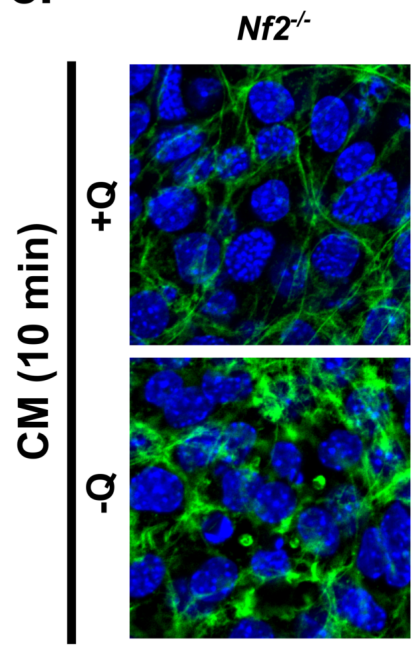

F-actin/DAPI

f.

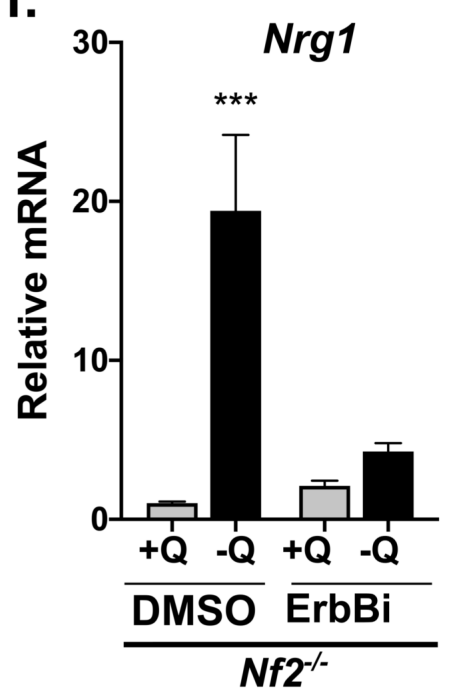

Chiasson-MacKenzie_Figure5

e.

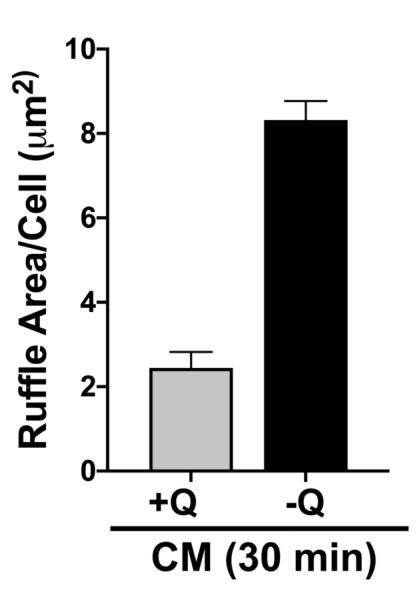

g.

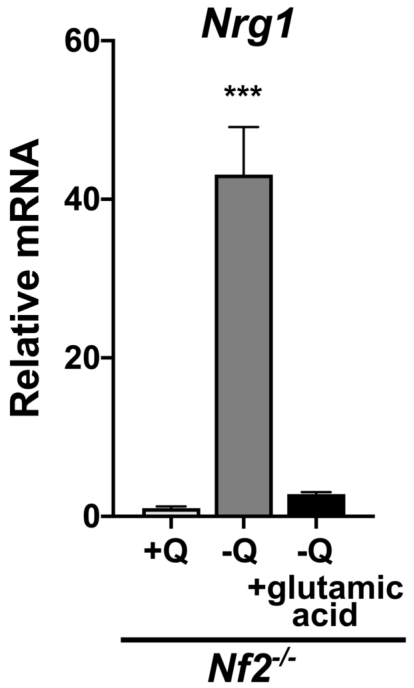

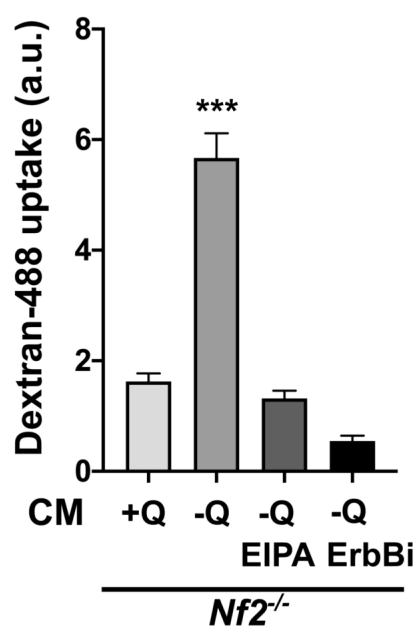


Figure 5: $\mathrm{Nf2}^{-/-} \mathrm{SCs}$ enact a distinct feedforward autocrine Nrg1 signaling program upon nutrient deprivation. A. Macropinocytic dextran-488 uptake in WT and Nf2-- SCs in response to $24 \mathrm{~h}$ glutamine deprivation with and without treatment with $2 \mu \mathrm{M}$ ErbBi. Bars represent mean +/- SEM for $n \geq 20$ cells per condition. $N=3$ independent experiments. B. mRNA expression of Nrg1 levels in WT and Nf2-- SCs grown for $24 \mathrm{~h}$ in complete medium (C), Nrg1-deprived medium (-Nrg), or glutamine-deprived medium (-Q). Data is presented as mean $+/-$ SEM relative to WT mRNA levels. $\mathrm{N}=3$ independent experiments. C. Confocal images of F-actin (green) depicting cortical ruffling in $\mathrm{Nf}^{-/-}$SCs stimulated for $10 \mathrm{~min}$ with conditioned medium from glutamine-deprived (-Q) but not glutamine replete (+Q) Nf2/- SCs. D. Quantitation of cortical ruffling from cells in (C). Bars represent mean + -- SEM for $n \geq 20$ cells per condition. $N=3$ independent experiments. E. Dextran-488 uptake in $\mathrm{Nf2}^{-/-} \mathrm{SCs}$ stimulated for 30 min with conditioned medium from glutamine-deprived (-Q) Nf2-/- cells treated with vehicle (DMSO), 50 $\mu \mathrm{M}$ EIPA, or $2 \mu \mathrm{M}$ ErbBi. Bars represent mean $+/-$ SEM for $\mathrm{n} \geq 20$ cells per condition. $\mathrm{N}=3$ independent experiments. F. Levels of Nrg1 mRNA expression in glutamine-deprived (-Q) Nf2-/SCs treated with vehicle (DMSO) or $2 \mu \mathrm{M}$ ErbBi. Data is presented as mean +/- SEM relative to $+\mathrm{Q}$, DMSO mRNA levels, $\mathrm{N}=3$ independent experiments. G. mRNA expression of Nrg1 levels in glutamine-deprived (-Q) Nf2-- SCs with or without addition of $10 \mathrm{mM} \mathrm{L-glutamic}$ acid. Bars represent mean $+/$ - SEM relative to $+Q$, DMSO mRNA levels, $N=2$ independent experiments. $P$ values were calculated with unpaired two-tailed Student's t-test. ${ }^{* *} p<0.001$. Scale bars $=10 \mu \mathrm{m}$. 


\section{Chiasson-MacKenzie_Figure6}

a.

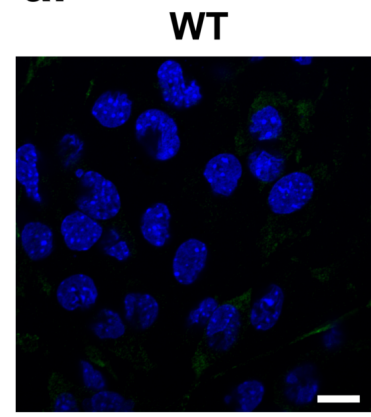

pS6/DAPI

d.

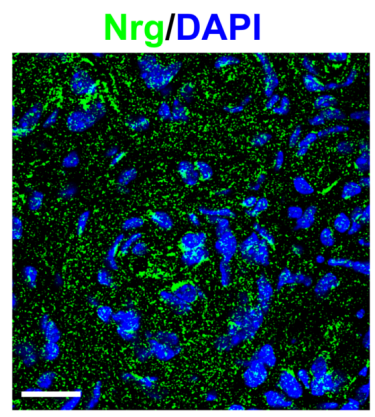

f.

g.

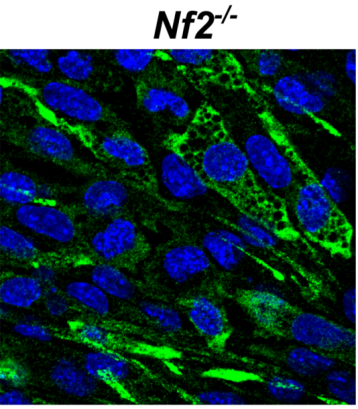

b.

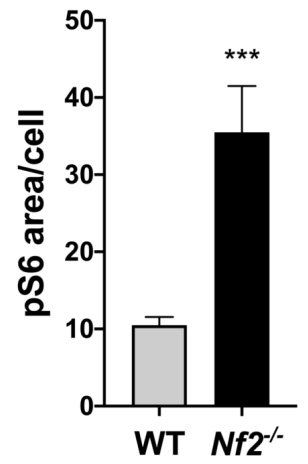

e.
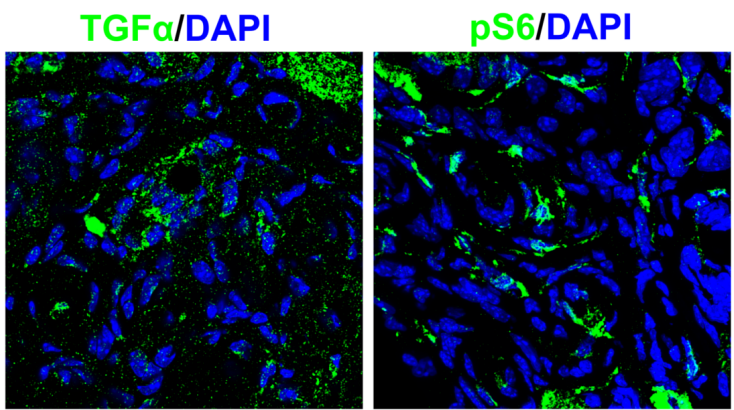

h.

Nrg1
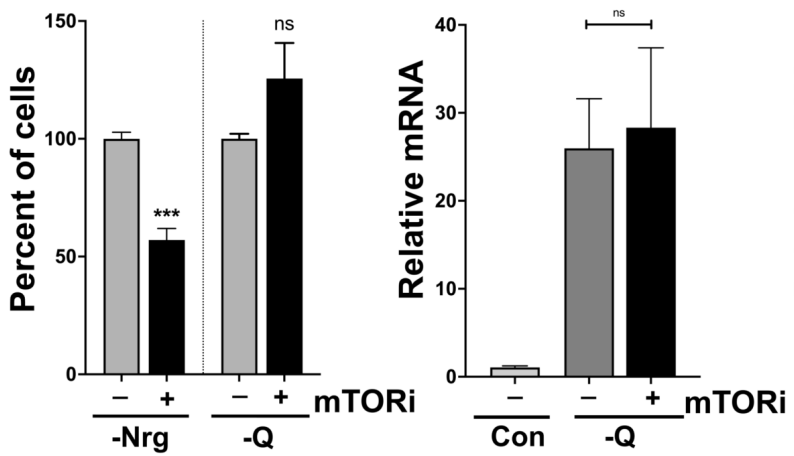

c.

Nrg1

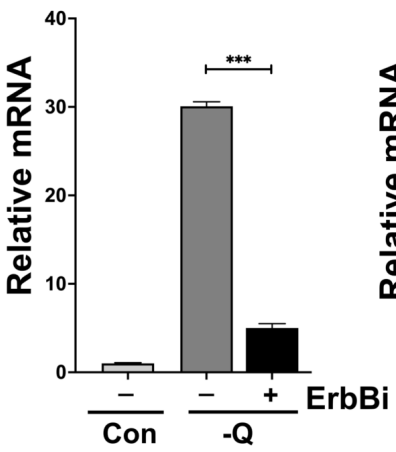

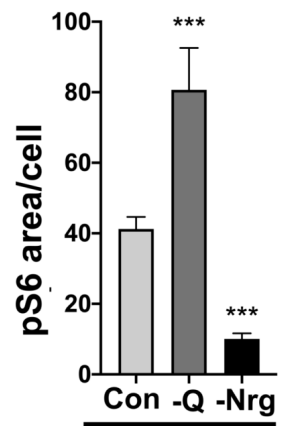

Conditioned Media

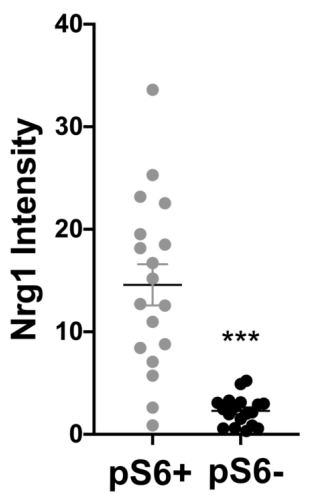

Tgfa

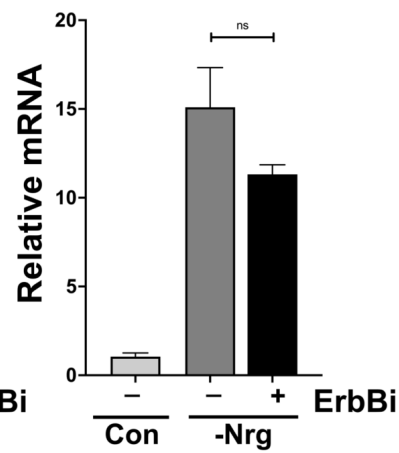


Figure 6. Heterogeneous mTOR activation and drug sensitivity A. Confocal images depicting the dramatic induction of pS6 (green) in Nrg1/glutamine-deprived $\mathrm{Nf}^{-/-} \mathrm{SCs}$ but not WT SCs. Scale bar=10 $\mu \mathrm{m}$. B. Quantitation of pS6 levels in cells in (A) as measured in threshold confocal images as the ratio between pS6 area and total cell area. Bars represent mean $+/$ - SEM in $n \geq 10$ cells per condition. $N=3$ independent experiments. C. Quantitation of pS6 levels in Nf2--- SCs treated with conditioned medium from glutamine- (-Q) or Nrg1-deprived $N f 2^{-/-}$SCs. Bars represent mean $+/-$SEM in $\mathrm{n} \geq 10$ cells per condition. $\mathrm{N}=3$ independent experiments. D. Confocal images of tissue from DRG lesions in a Postn-Cre;Nf2 ${ }^{\text {floxfllox }}$ mouse stained for Nrg1, TGF $\alpha$, or pS6. Scale bar=20 $\mu$ m. E. Graph depicting Nrg1 intensity in cells in staining positively or negatively for pS6 in tissues double-labeled for Nrg1 and pS6. Lines represent mean +/- SEM for $n=18$ cells per condition. F. Quantitation of cell viability of $N f 2^{-/-}$SCs deprived of Nrg1 or glutamine upon treatment with mTORi (100 nM, 72h). Bars represent mean +/- SEM percentage of cells compared to vehicle treatment. $\mathrm{N}=3$ independent experiments. G. Nrg1 mRNA expression levels in glutamine-deprived $\mathrm{Nf}^{-/-} \mathrm{SCs}$ treated with vehicle (DMSO) or $100 \mathrm{nM}$ mTORi. Data is presented as mean +/- SEM relative to $+Q$, DMSO mRNA levels, $N=3$ independent experiments. H. Levels of $\mathrm{Nrg} 1 \mathrm{mRNA}$ expression in glutamine-deprived $\mathrm{Nf}^{-{ }^{-}} \mathrm{SCs}$ or Tgfa mRNA expression in Nrg-deprived $\mathrm{Nf2}^{-/-}$SCs treated with vehicle (DMSO) or $2 \mu \mathrm{M}$ ErbBi. Data is presented as mean +/- SEM relative to control, DMSO mRNA levels, $N=3$ independent experiments. P values were calculated with unpaired two-tailed Student's t-test. ${ }^{* * *} p<0.001$ 


\section{Chiasson-MacKenzie_Figure7}

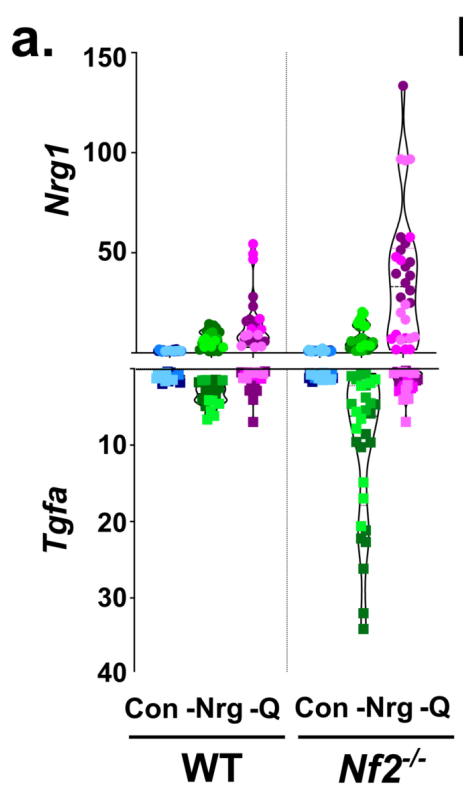

a.

C.

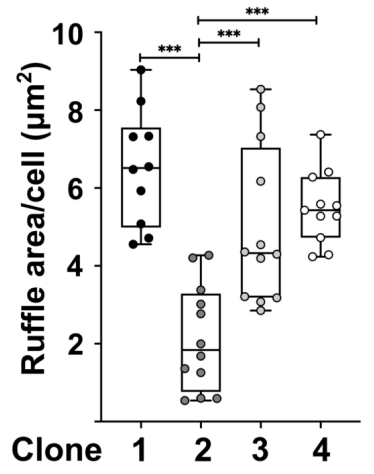

f.

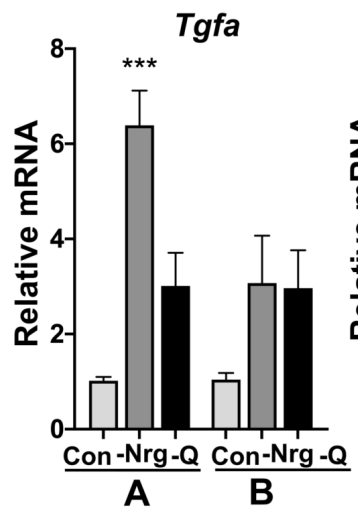

b.

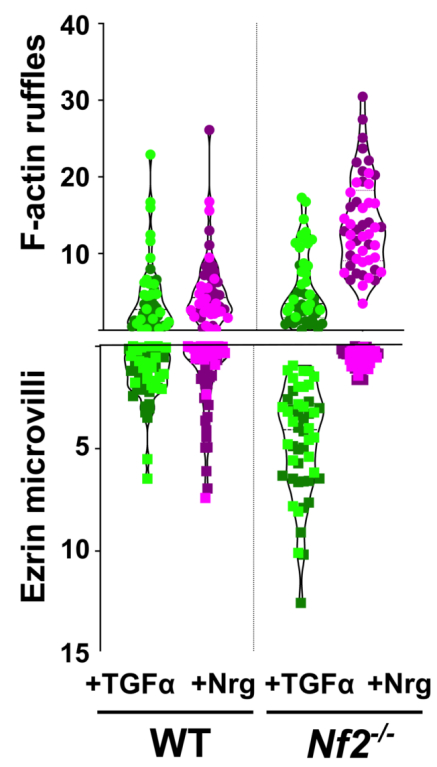

d.

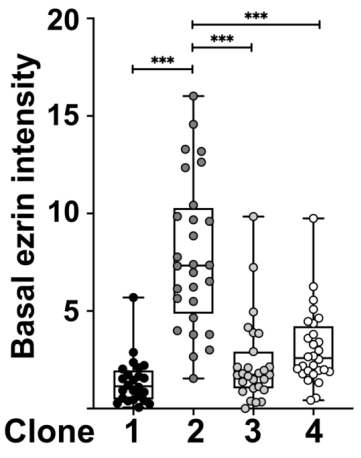

e.
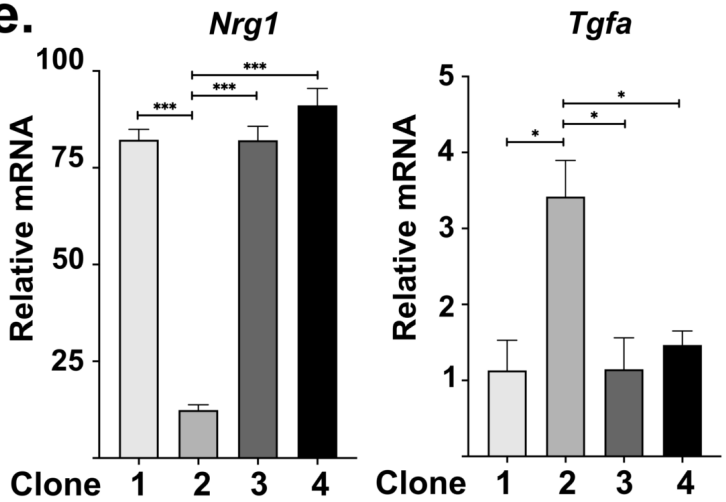

g. TGFa/DAPI
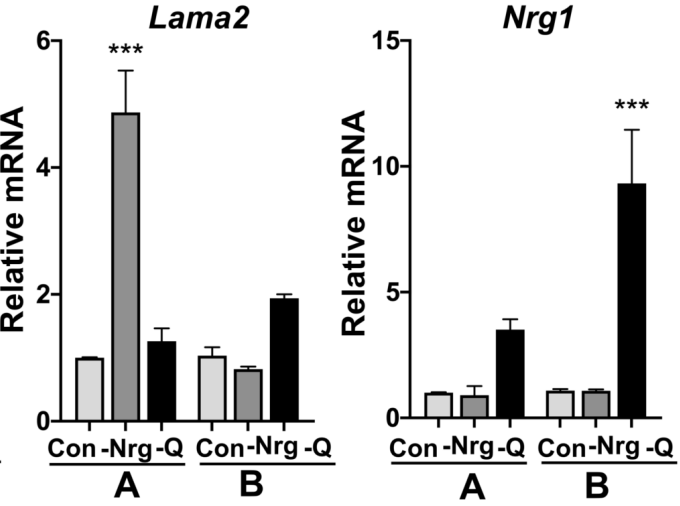

竞

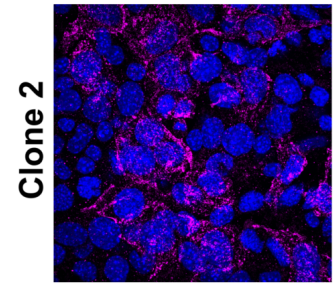


Figure 7. Self-generated heterogeneity. A. Summary graphs depicting mRNA levels of Nrg1 and Tgfa in three independently generated $\mathrm{Nf}^{-/-}$and WT SC lines grown under steady-state conditions (blue) or deprived of Nrg1 (green) or glutamine (magenta) for $24 \mathrm{~h}$. Data is presented as a violin plot where each dot represents fold change of mRNA relative to steady state conditions of the same genotype of an individual replicate and each SC line is represented by a different shade of color (light, medium, or dark). B. Summary graphs depicting changes in polarized cytoskeletal organization in $\mathrm{Nf}^{--}$and WT SC lines stimulated with either TGF $\alpha$ (green) or Nrg1 (magenta) for $10 \mathrm{~min}$. Data is presented as a violin plot where each dot represents the ratio between actin or ezrin area and total cell area in a single cell in threshold images and each SC line is represented by a different shade of color (medium or dark). C. Quantitation of cortical actin ruffling in four independently generated clonal populations of $\mathrm{Nf}^{-/-} \mathrm{SCs}$. Box and whisker plot shows minimum to maximum values of actin area with each dot representing a $63 x$ field of view. $n \geq 10$ fields per clone. D. Quantitation of basal ezrin intensity in clonal populations of $N f 2^{-/-}$ SCs. Box and whisker plot shows minimum to maximum values of the ratio of ezrin area to total cell area with each dot representing a single cell in a threshold image. $n=25$ cells per clone. $\mathrm{N}=3$ independent experiments E. mRNA expression of Nrg1 and Tgfa levels in clonal populations of $N f 2^{---}$SCs relative to steady state parental $N f 2^{-/-}$SCs. $\mathrm{N}=2$ independent experiments. F. mRNA expression of Tgfa, Lama2, and Nrg1 in schwannoma A and schwannoma B cells under steady state (Con), Nrg1-, or glutamine-deprived conditions (-Q). Data is presented as mean +/- SEM relative to steady state mRNA levels in schwannoma $A$ cells, $\mathrm{N}=3$ independent experiments. G. Confocal images depicting TGF $\alpha$ (magenta) distribution in parental $\mathrm{Nf}^{-/-} \mathrm{SCs}$ following $24 \mathrm{~h}$ Nrg1 deprivation and clonal $\mathrm{Nf}^{-/-} \mathrm{SCs}$ after long term Nrg1 deprivation. Scale bar=10 $\mu \mathrm{m}$. P values were calculated with unpaired two-tailed Student's ttest. ${ }^{*} p<0.05,{ }^{* * *} p<0.001$ 
Chiasson-MacKenzie_Figure8

a.

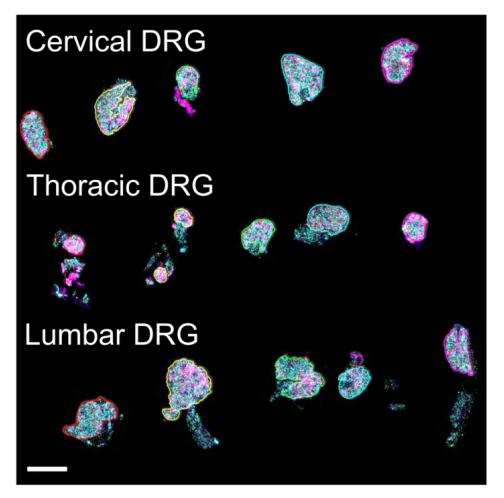

C.

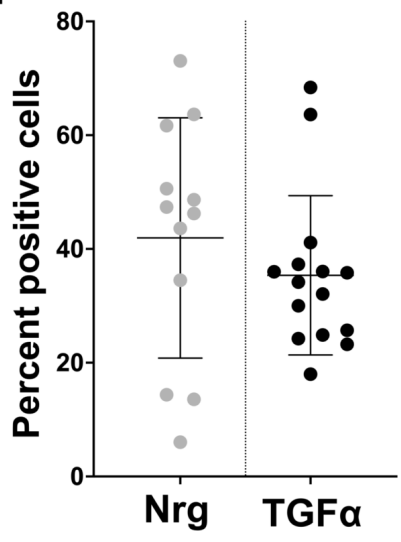

d.
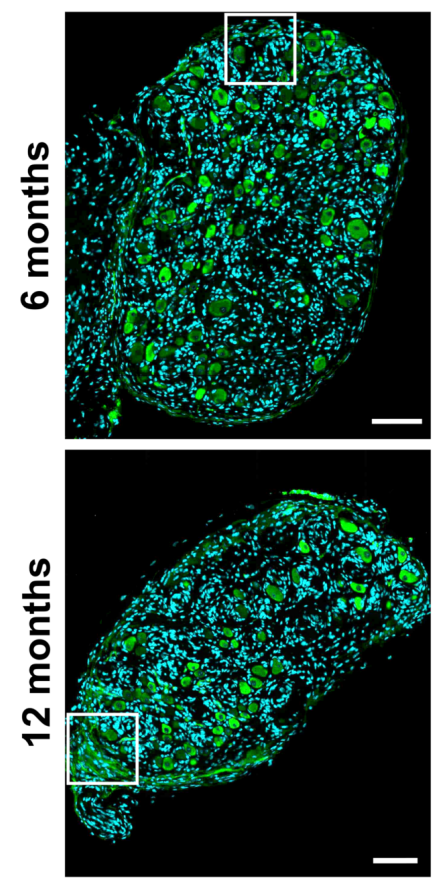

TGFa/DAPI b.

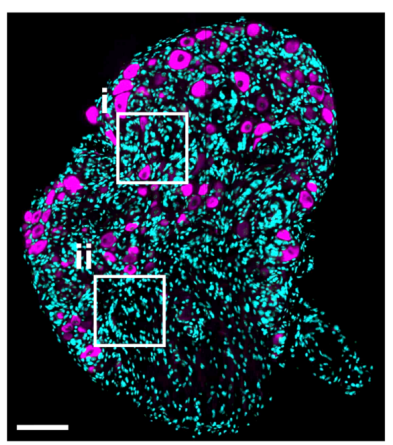

Nrg1/DAPI

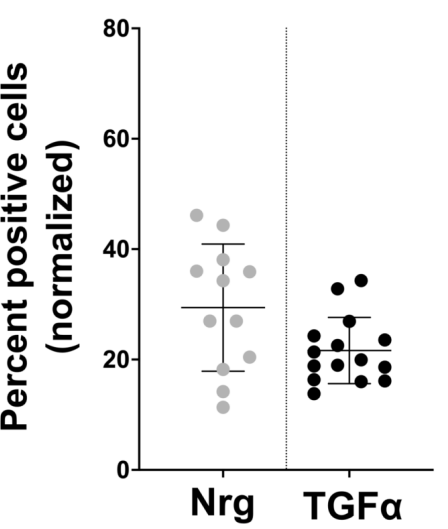

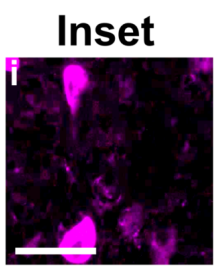

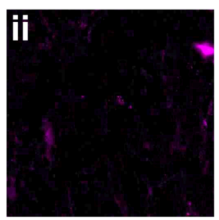

Proximity analysis

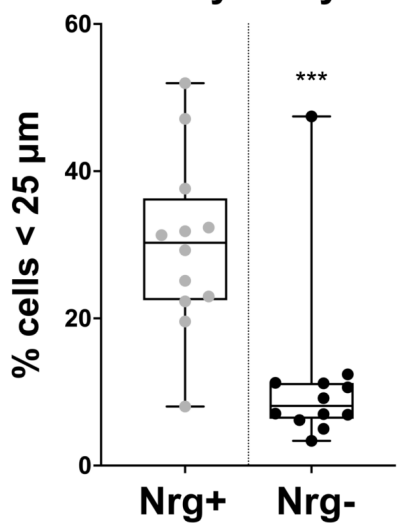

\section{Cluster Annotation}

e.
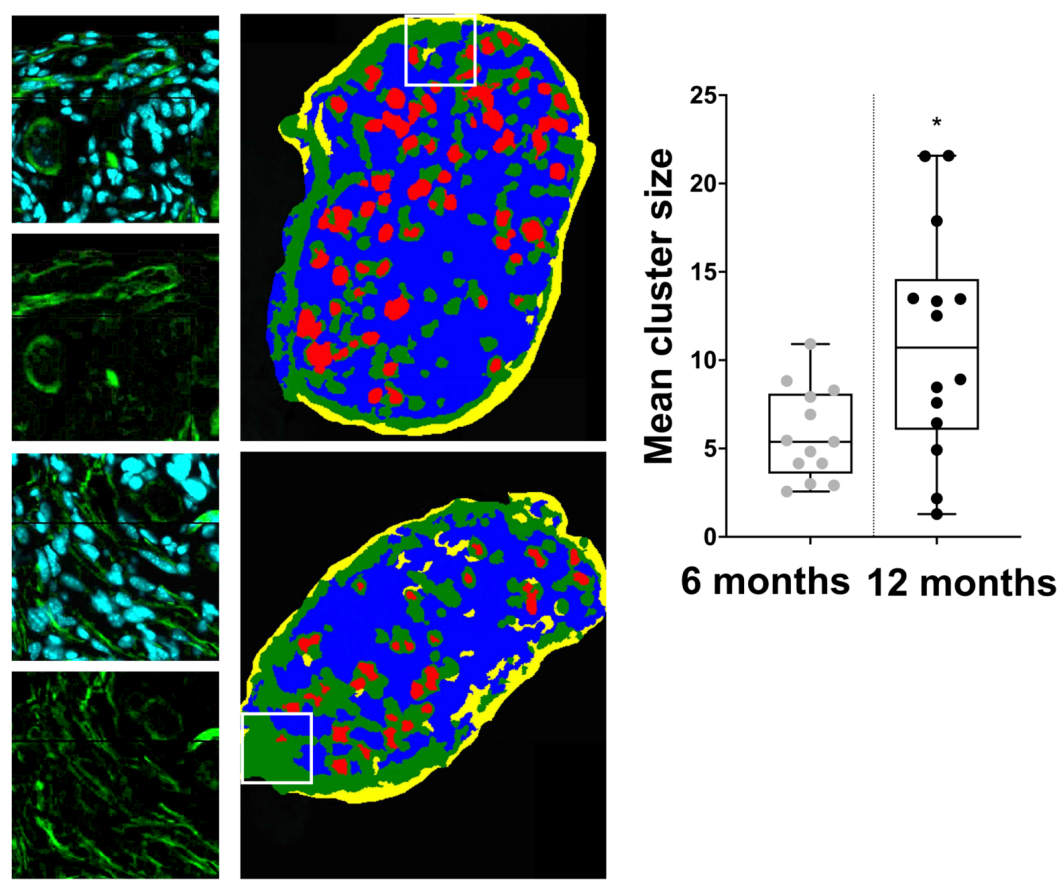

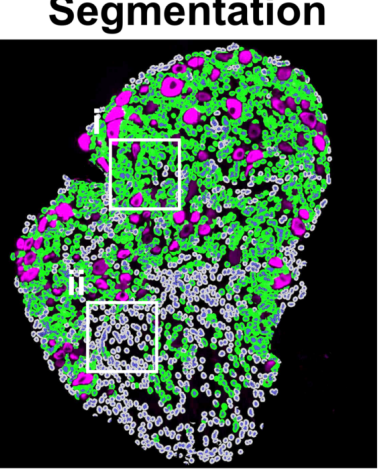


Figure 8. Quantitative analysis of self-generated heterogeneity in schwannoma tissue. A. Representative full slide 40x scan of the array of DRGs from a 6 month-old Postn-Cre;Nf2 ${ }^{\text {floxflox }}$ mouse stained for TGF $\alpha$ (magenta) and DAPI (cyan). Scale bar=1 mm. B. Left, Image of a single DRG lesion from a 6 month-old Postn-Cre;Nf2 ${ }^{\text {floxflox }}$ mouse stained for Nrg1 (magenta) and DAPI (cyan). Inset depicts Nrg1 expression close to (i) and far away from (ii) DRG soma. Right, Overlay of cell segmentation algorithm analysis depicting Nrg1 expression (green) in a representative DRG lesion. Scale bar=100 $\mu \mathrm{m}$. C. Left, Graph showing percentage of cells positive for Nrg1 or TGF $\alpha$ in 6 month-old DRG lesions. Each dot represents one DRG lesion. (middle) graph of percentage of Nrg1 and TGF $\alpha$ positive cells normalized to the area of DRG soma in each lesion. Right, Graph showing results of proximity analysis measuring the percentage of Nrg1+ and Nrg1- cells located within $25 \mu \mathrm{m}$ of DRG soma in 6 month old DRG lesions. D. Left, Images of single DRG lesions from a 6 month-old and 12 month old PostnCre;Nf2 $2^{\text {flox/flox }}$ mouse stained for TGF $\alpha$ (green) and DAPI (cyan). Inset depicts TGF $\alpha$ clustering at the periphery of DRG lesions. Right, Overlay depicting results of Random Forest machine learning algorithm to classify DRG soma (red), TGF $\alpha$ positive cells (green), TGF $\alpha$ negative cells (blue), and unclassified area (yellow). F. Quantitation of mean cluster size of TGF $\alpha$ positive cells in 6 month and 12 month DRG lesions. Each dot represents one DRG lesion. Scale bar=100 $\mu \mathrm{m} . \mathrm{P}$ values were calculated with unpaired two-tailed Student's t-test. ${ }^{*} p<0.05,{ }^{* * *} p<0.001$. 